\title{
méthode de prévision des pressions interstitielles
}

\author{
par \\ M. Boulon \\ Maître Assistant \\ F. Darve \\ Maitre Assistant \\ E. Flavigny \\ Assistant \\ M. Forgue \\ Chercheur \\ Institut de Mécanique \\ Université Scientifique et Médicale de Grenoble
}

Conférence devant le Comité Français de la mécanique des sols et des fondations, le 19 février 1979.

Introduction

Deux classes d'essais triaxiaux sont utilisés par les laboratoires d'essais de Mécanique des Sols : les essais drainés et les essais non-drainés. Dans les premiers la phase liquide est libre de se mouvoir par rapport au squelette solide, tandis que dans les seconds on a un couplage entre phase liquide et phase solide et création d'une pression interstitielle: on impose alors une sollicitation mécanique mixte en obligeant la phase liquide et la phase solide à réagir ensemble. Dans le cas où il est parfaitement saturé, le sol devient incompressible : nous nous restreindrons à ce cas.

L'exploitation des essais C.U. (pour " consolidatedundrained ", selon la terminologie habituelle) se fait généralement en contraintes effectives c'est-à-dire en écrivant :

$$
\left\{\begin{array}{l}
\sigma_{n}^{\prime}=\sigma_{n}-u \\
\tau^{\prime}=\tau
\end{array}\right.
$$

qui traduit le principe de Terzaghi. On peut ainsi déterminer ' $C^{\prime}$ et $\Phi^{\prime}$ cohésion et angle de frottement effectifs, qui sont voisins des cohésion et angle de frottement mesurés sur chemins drainés.

Pour les matériaux argileux, les essais C.U., plus rapides à effectuer sont généralement préférés aux essais C.D. ( " consolidated drained »). Au contraire, les essais C.U. sur sables sont plus rarement réalisés sauf lorsque l'on s'intéresse à l'étude de la liquéfaction. Cependant les véritables propriétés d'un sol sont celles mesurées sur chemin drainé comme le note Kérisel [15]

C'est à partir d'une loi rhéologique incrémentale déterminée sur chemin drainé, aussi bien pour matériaux sableux qu'argileux, et dont nous rappelons les principaux traits dans la première partie que nous simulerons le comportement sur chemin non-drainé de sables et argiles en homogène (essai triaxial) et hétérogène (ouvrages). Des simulations de ce type ont été réalisées avec d'autres lois rhéologiques : les résultats donnés par l'élasticité linéaire sont classiques en homogène; Lade [16] et Matsuoka [19] fournissent des exemples comparables aux nôtres de passages de propriétés mécaniques drainées aux propriétés mécaniques non-drainées. En hétérogène (champ de pressions interstitielles hétérogène), les calculs réalisés rigoureusement en contraintes effectives avec propriétés mécaniques intergranulaires sont très peu nombreux.

La première partie de cet article concerne les résultats obtenus en champ homogène, tandis que la seconde partie se rapporte aux champs hétérogènes.

\section{Utilisation de la loi rhéologique en laboratoire}

\subsection{La loi rhéologique utilisée}

Soit $F$ la fonction décrivant les propriétés mécaniques incrémentales d'un échantillon de sol à l'instant actuel. Cette fonction $\mathrm{F}$ dépend de l'histoire antérieure du matériau et relie, à l'instant actuel, la petite déformation pure $\mathrm{d} \varepsilon$, l'incrément de contrainte do (pris en axes co-rotationnels) et l'incrément de temps $\mathrm{dt}$ :

$F(d \varepsilon, d \sigma, d t)=0$

Pour un matériau, comme le sol, comportant des irréversibilités plastiques, nous savons bien que cette fonction $F$ n'est pas linéaire. Restreignons cette étude au cas des matériaux non-visqueux (l'incrément de temps n'intervient plus) et considérons : $d \varepsilon=G(d \sigma)$. La fonction $\mathrm{G}$ est homogène, d'ordre 1, en d $\sigma$, (par indépendance du comportement par rapport aux vitesses de sollicitation), mais elle n'est pas linéaire puisque $\mathrm{G}(-\mathrm{d \sigma} \sigma)$ n'est pas égal à : - $\mathrm{G}(\mathrm{d \sigma})$ du fait de l'existence de déformations irréversibles. Cette non-linéarité est particulièrement importante à décrire, puisque c'est elle qui permet de prendre en compte fondamentalement le comportement plastique.

Historiquement, les premières tentatives pour décrire cette non-linéarité ont consisté à prendre deux fonctions linéaires distinctes $G_{1}$ et $G_{2}$ suivant que la sollicitation incrémentale appartenait à un domaine ou un autre. C'est par exemple le cas, à la fois des modèles élastiques bilinéaires (Duncan, Chang [11]) et des lois élasto-plastiques classiques (Nayak, Zienkiewicz [24]) qui distinguent $\mathrm{G}_{1}$ de $\mathrm{G}_{2}$ par un critère de charge-décharge ou de dissipation positive ou négative. II nous semble que l'introduction récente d'un double potentiel plastique (Maier et Hueckel [18], Lade [17]) ou d'une règle d'écoulement plastique variant avec le chemin de sollicitation suivi (Mroz [23], 
Prevost [26]) ne lève que partiellement les problèmes soulevés par la description de la non-linéarité incrémentale par uniquement deux déterminations $G_{1}$ et $G_{2}$, même si ces développements améliorent la souplesse de l'élastoplasticité traditionnelle.

II parait nécessaire à la fois pour des raisons physiques et pour une utilisation numérique plus commode dans des programmes " éléments finis \# de décrire cette non-linéarité de manière continue.

La loi rhéologique, que nous avons nous-mêmes développée (nous l'appellerons le modèle Linol : Loi Incrémentale Non-Linéaire), était caractérisée dans sa forme initiale (Darve [7], Boulon et al [2]) par une matrice $M$ symétrique $(\mathrm{d} \varepsilon=\mathrm{Md \sigma})$ pouvant prendre 8 déterminations différentes suivant la direction de la sollicitation incrémentale (Linol -S). Pour rendre la loi continue au passage d'une détermination à une autre, nous avons été amenés à considérer une matrice $M$ non-symétrique (Darve et al [9]) (Linol - N.S.) puis à généraliser nos formulations des sables aux argiles (Flavigny [12], Darve et al [10]) (Linol - N.S.A.). enfin à prendre en compte un terme visqueux (Darve [7], Vuaillat [30]) (Linol - N.S., A.V.).

Le dernier développement (Chambon et al [4]) porte sur l'introduction d'une variation continue de la matrice $M$ avec la direction de la sollicitation incrémentale (Linol - N.S.C.).

Dans cet article, les résultats concernant les calculs d'essais homogènes utilisent le modèle Linol - N.S., tandis que les calculs menés avec la méthode des éléments finis font appel à Linol - S qui nécessite pour être utilisé l'adjonction de deux algorithmes: l'un de prévision de la zone tensorielle de fonctionnement, et le second de passage à l'état non-drainé. Le lecteur trouvera ces différentes lois explicitées dans les références citées précédemment. Nous nous bornerons ici à un rappel très succinct de Linol - N.S.

Partant de la formulation générale : $d \varepsilon_{\alpha}=M_{\alpha \beta} d \sigma_{\beta}(\alpha, \beta=$ $1, \ldots, 6)$ nous faisons deux hypothèses :

1 - I'anisotropie induite par le chemin de sollicitation antérieur est approchée comme étant de l'orthotropie. La loi incrémentale est donc supposée orthotrope

2 - la non-linéarité incrémentale est décrite par 8 déterminations distinctes pour la matrice $\mathrm{M}$, associées à 8 «zones tensorielles » dans l'espace des incréments de contrainte, ces 8 zones étant constituées par les 8 huitièmes d'espace batis sur les trois vecteurs orthogonaux portant $d \sigma_{11}, d \sigma_{22}$ et $d \sigma_{33}$. Ces 8 applications linéaires sont alors entièrement définies par la connaissance du comportement du matériau sur les 6 demi-axes $\left( \pm d \sigma_{11}, d \sigma_{22}=0\right.$, $\left.d \sigma_{33}=0\right),\left(d \sigma_{11}=0, \pm d \sigma_{22} d \sigma_{33}=0\right),\left(d \sigma_{11}=0, d \sigma_{22}=0\right.$, $\pm d \sigma_{33}$.

La loi prend la forme suivante :

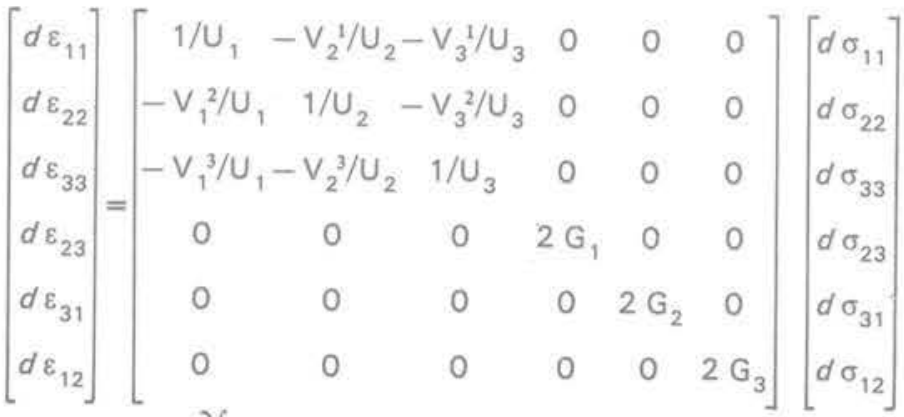

$$
\begin{aligned}
& \text { où : } U_{k}=\left(\frac{\partial f}{\partial \varepsilon_{k}, \sigma_{j}, \sigma_{1}} \sigma_{\gamma}, \sigma_{1}\right. \text { constants, } \\
& \mathrm{V}_{\mathrm{k}}^{\prime}=-\left(\frac{\partial g}{\sigma \varepsilon_{\mathrm{k}}}\right)_{\sigma_{j}, \sigma_{i}} \quad \mathrm{~V}_{\mathrm{k}}^{\prime}=-\left(\frac{\partial h}{\sigma \varepsilon_{\mathrm{k}}}\right)_{\sigma_{j}, \sigma_{l}}
\end{aligned}
$$

avec : $\sigma_{\mathrm{k}}=\mathrm{f}\left(\varepsilon_{\mathrm{k}^{\prime}}, \sigma_{\mathrm{j}^{\prime}} \sigma_{l}\right) ; \varepsilon_{\mathrm{j}}=\mathrm{g}\left(\varepsilon_{\mathrm{k}^{\prime}} \sigma_{\mathrm{j}}, \sigma_{l}\right) ; \varepsilon_{l}=\mathrm{h}\left(\varepsilon_{\mathrm{k}^{\prime}} \sigma_{\mathrm{j}^{\prime}} \sigma_{l}\right)$ $\left(\sigma_{1}\right.$ et $\sigma_{1}$ constants $)(k, j, I=1,2,3 ; k \neq j \neq 1)$,

enfin: $G_{1}=\frac{\sqrt{\left(U_{2}\right)^{2}+\left(U_{3}\right)^{2}}}{U_{2} U_{3} \sqrt{2}}+\frac{U_{2}\left(V_{3}{ }^{2}-1\right)+U_{3}\left(V_{2}{ }^{3}-1\right)}{4 U_{2} U_{3}}$

$\mathrm{G}_{2}$ et $\mathrm{G}_{3}$ se déduisant de $\mathrm{G}_{1}$ par permutation circulaire sur les indices.

Nous nous donnons les 3 familles de fonctions $f, g$, $h$, en compression $\left(U_{k} d \sigma_{k k}>0\right)$ et en extension $\left(U_{k} d \sigma_{k k}<0\right)$, sous forme analytique explicite, à caler sur les courbes expérimentales du sol considéré (ce calage fournit les paramètres du sol).

II reste à vérifier la condition essentielle de continuité au passage d'une zone à une zone adjacente: sur la matrice le changement de zone se traduit par la modification des trois termes d'une colonne, mais précisément de la colonne qui, pour le calcul de l'incrément de réponse, est multipliée par l'élément du vecteur incrément de contrainte qui s'annule au changement de zone. La continuité est ainsi assurée dans le cas d'une matrice $M$ non-symétrique.

Pour prévoir le comportement d'un échantillon de sol pendant un essai triaxial de révolution non drainé, nous avons en champ homogène :

$\mathrm{d} \varepsilon_{2}=\mathrm{d} \varepsilon_{3}$

$\mathrm{d} \sigma_{2}^{\prime}=\mathrm{d} \sigma_{3}^{\prime}$

Si nous nous plaçons dans le cadre d'un essai sur un échantillon parfaitement saturé, on peut écrire la non-variation de volume :

$\mathrm{d} \varepsilon_{1}+2 \mathrm{~d} \varepsilon_{3}=\mathrm{d} \varepsilon_{\mathrm{v}}=0$

La condition (2) peut être modifiée pour tenir compte d'une légère variation de volume au cours de l'essai, due soit à une saturation imparfaite soit à la variation de volume introduite par la capote entourant l'échantillon (cf Flavigny [12]).

Si l'on se fixe arbitrairement la valeur de l'incrément de déformation axiale $d \varepsilon_{1}$, la condition (2) fournit $d \varepsilon_{3}$, incrément de déformation latérale. La résolution de

$\mathrm{d} \varepsilon_{\alpha}=\mathrm{M}_{\alpha \beta} \mathrm{d} \sigma_{\beta}$

permet alors de calculer les incréments de contraintes intergranulaires $\mathrm{d \sigma} \sigma_{1}$ et $\mathrm{d} \sigma_{3}$.

Pour un essai triaxial classique de compression, $\sigma_{3}$ contrainte latérale totale est fixe et l'on a

$\sigma_{3}^{\prime}=\sigma_{3}-u$

où u représente la pression interstitielle.

Par conséquent :

$\mathrm{d \sigma}_{3}=-\mathrm{du}$

La relation (6) permet alors le calcul de la pression interstitielle.

II faut noter que l'on ne simule pas en fait un essai triaxial " non drainé " classique mais un essai « à pression interstitielle nulle »: les deux essais sont équivalents et l'on trouve par exemple chez Mir Emerati [21] ou Anquetil [1] une comparaison des deux types d'essais.

Pour présenter les résultats de cette intégration de la loi rhéologique incrémentale nous fournirons sur chaque exemple trois figures $a, b, c$ :

La figure a sera une courbe "effort-déformation » : $\sigma_{1}-\sigma_{3}$ fonction de $\varepsilon_{1}$.

La figure b sera la réponse en pression interstitielle.

Enfin la figure c sera le chemin de contrainte effective (généralement dans le plan

$$
\left.\mathrm{p}=\frac{\sigma_{1}^{\prime}+2 \sigma_{3}^{\prime}}{3} \mathrm{q}=\sigma_{1}-\sigma_{3}^{\prime}\right):
$$

ce chemin se termine sur la surface limite de plasticité. 

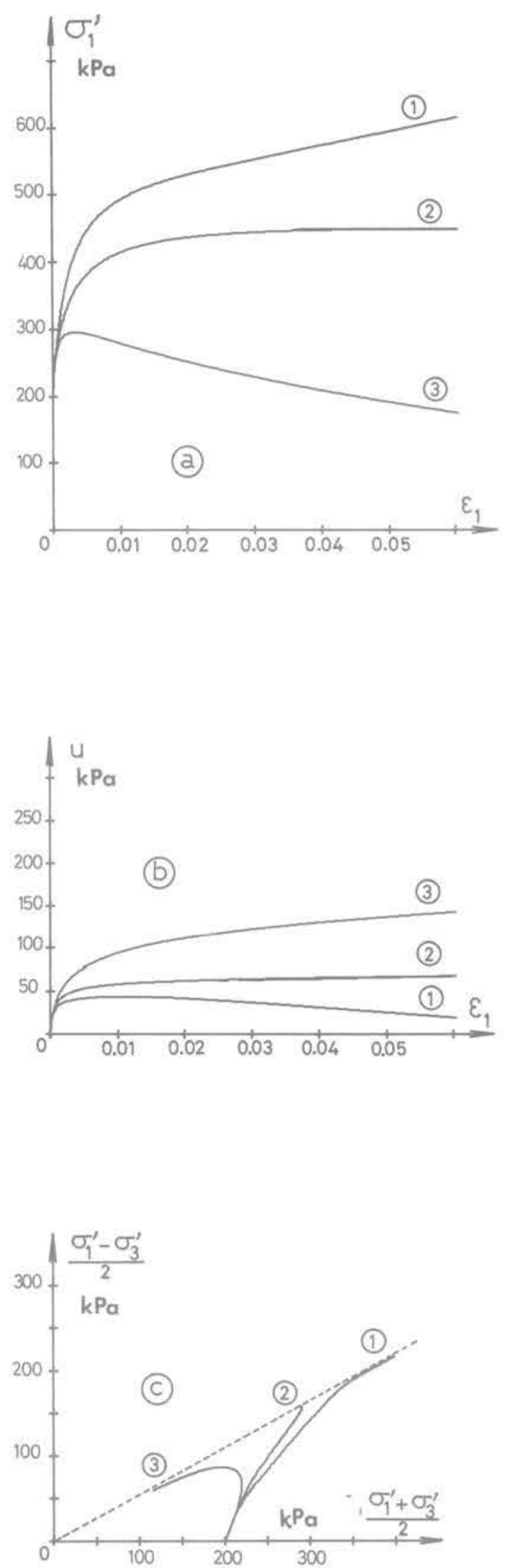

(1) $e_{0}=0.788$

(2) 0.823

(3) 0.86

Fig. 1-1 Simulation d'un essai C.U sur le sable de Monterey

\subsection{L'essai consolidé non-drainé sur sable}

Dans l'essai C.U. sur sable, l'échantillon est préparé à un indice des vides donné puis saturé. Après consolidation sous la pression d'écrasement le drainage est fermé et l'échantillon écrasé : ce chemin est moins employé que le chemin drainé.

La figure (1.1 a b b c) présente les résultats de simulation numérique sur le sable de Monterey pour lequel la détermination de la loi rhéologique avait été faite par Darve et al [8].

Les courbes notées 1 correspondent à l'indice des vides de 0,78 : le sable pour la pression latérale choisie est légèrement dilatant et le chemin de contrainte obtenu remonte la surface limite : ceci correspond à une pression interstitielle qui décroit. Notons que sur ce type de chemin - et dans le domaine de déformation étudié - le critère $\left(\sigma_{1} / \sigma_{3}\right)$ max est réalisé sans que l'écoulement plastique du matériau ne soit atteint: l'état de contrainte continue à évoluer.

Les courbes notées 2 sont celles d'un sable lâche pour lequel l'écoulement plastique du matériau est atteint.

Enfin les courbes 3 correspondent au sable le plus lâche : dans ce cas on note un pic (fig. 1.1 a) qui n'existe pas sur chemin drainé. La surface de plasticité $\left(\sigma_{1}^{\prime} / \sigma_{3}^{\prime}\right)$ max est atteinte après ce pic $\left(\sigma_{1}-\sigma_{3}\right)$ max.

A titre de comparaison nous présentons en figure (1.2) une série d'essais triaxiaux C.U. réalisés par Boutwell [3] et que nous avons retracés dans le plan $(p, q)$ : les différentes formes de chemins observés sur la figure (1.1 c) se retrouvent la aussi, notamment les chemins de contrainte correspondant aux indices des vides $e_{0}=0,78$ et $e_{0}=0,93$ qui sont analogues aux chemins 1 et 3 de la figure (1.1 c).

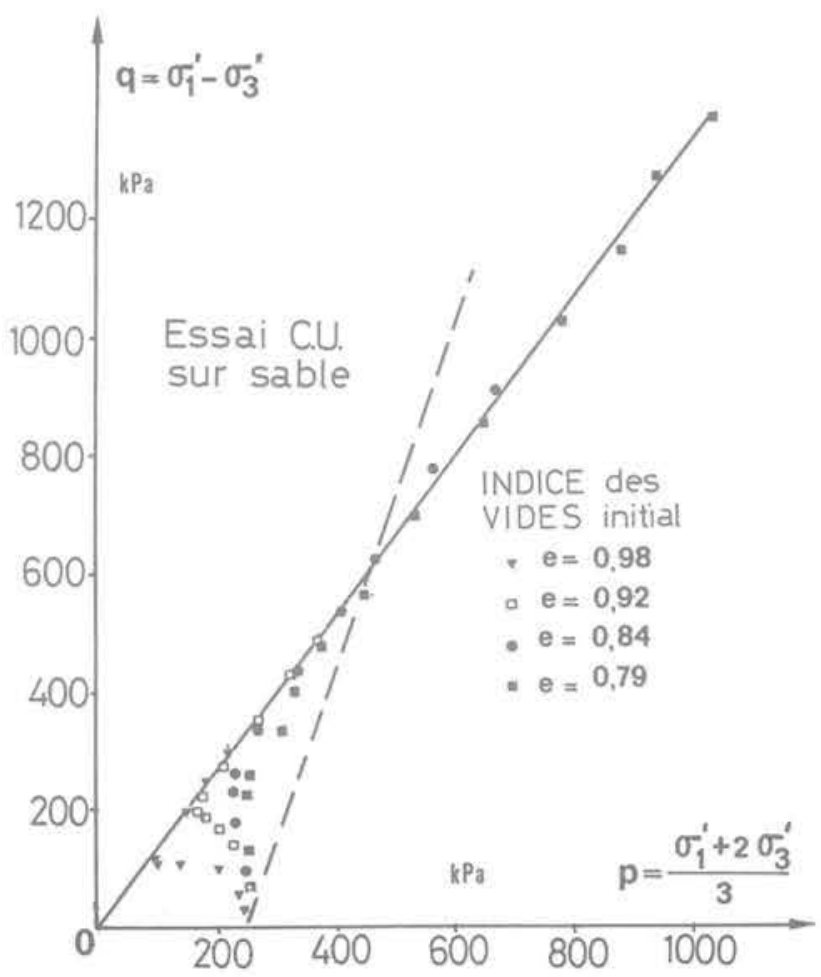

Fig. 1-2 Essais non-drainés (d'après Boutwell) 

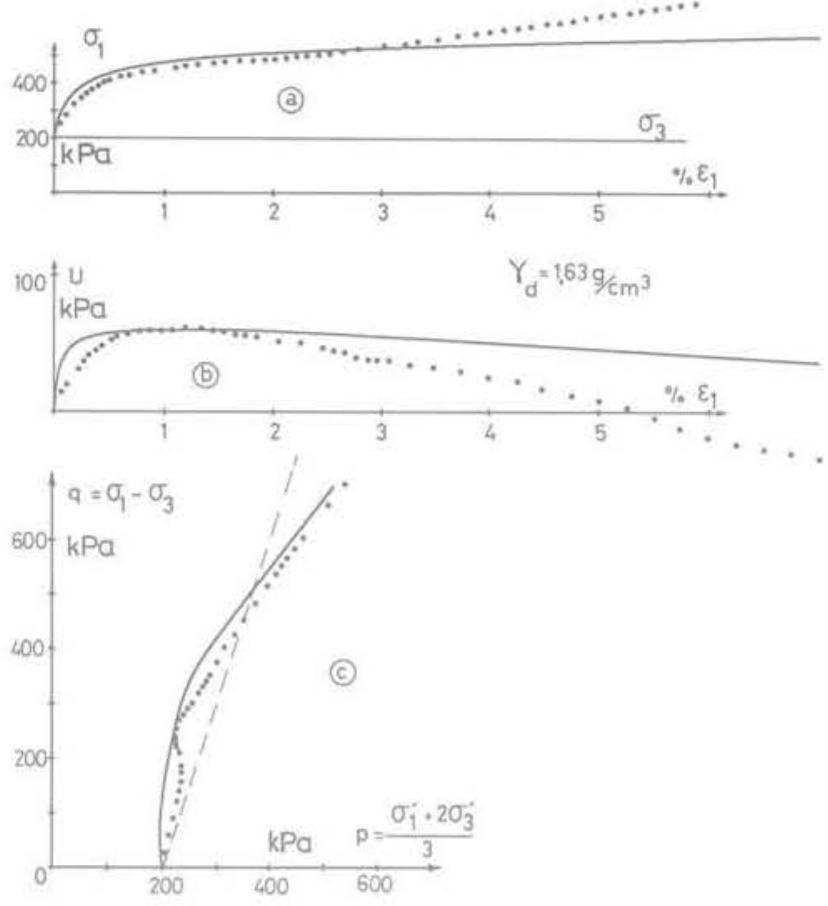

Fig. 1-3 Essai non-drainé sur le sable d'Hostun (points expérimentaux et courbes calculées)

Sur le sable d'Hostun, la figure (1.3 a, b, c) présente une comparaison entre les résultats d'un essai non-drainé (points expérimentaux) et la prédiction qui en a été faite à partir de la loi rhéologique drainée. II faut noter que les essais drainés avaient été faits avec du sable sec sur l'appareil «Phogadense » qui permet la mesure locale de densité tandis que l'essai non-drainé a été effectué sur un appareillage classique : la concordance obtenue est bonne bien qu'il faille remarquer que la décroissance prévue de la pression interstitielle est trop faible (fig. 1.3, b). Les chemins de contraintes obtenus (fig. $1.3, \mathrm{c}$ ) remontent tous deux la surface limite de plasticité : à la masse volumique sèche de $1,63 \mathrm{~g} / \mathrm{cm}^{3}$, ce sable est dilatant. En début d'essai la pression interstitielle prévue est plus faible que celle mesurée : il y a peut-être en début d'essai un mauvais centrage de l'échantillon. De plus les conditions aux limites dans les deux essais étaient légèrement différentes: l'appareil " Phogadense " était muni de rotules en tête et en pied tandis que l'appareillage classique n'en possédait pas.

Les résultats obtenus montrent que l'on peut prévoir la pression interstitielle lors d'un écrasement C.U. sur sable et méritent les remarques suivantes:

a) il n'y a eu aucun "calage " entre essai drainé et essai non-drainé

b) l'effet de la membrane (cf. Flavigny [12]) peut fausser la réponse en pression interstitielle

c) la diminution de la pression interstitielle qui se produit avec la remontée de la surface limite cause une désaturation progressive de l'échantillon (qui peut à l'extrême s'accompagner de la cavitation lorsque la pression interstitielle devient inférieure à $0,80 \mathrm{kPa}$ ).

\subsection{L'Essai consolidé non-drainé sur argile}

La détermination de la loi incrémentale (Loi Linol N.S.A.) de l'argile de La Roche Chalais a été effectuée aussi à partir d'essais drainés et est exposée par ailleurs (cf Flavigny [12] et Darve et al [11]) ; nous ne présenterons ici que la comparaison entre des essais C.U. de compression et d'extension et la simulation numérique qui peut en être faite avec la loi Linol N.S.A.
La figure (1.4 a, b, c) compare alors les courbes théoriques riques obtenues par intégration de la loi, avec les points expérimentaux mesurés dans trois essais consolidés nondrainés : deux essais sont des essais de compression sous consolidation de 200 et $400 \mathrm{kPa}$ (notés C.U. 2 et C.U. 4), un essai est fait en extension (E.U. 4). Les valeurs théoriques (trait continu) obtenues par intégration de la loi sont plus faibles que les valeurs expérimentales (points). Notons que l'inverse était observé sur les figures (1.3 a) et (1.3 b). En extension les variations de pression interstitielle sont très faibles, en accord avec de très faibles variations de volumes sur chemins drainés. L'angle de frottement effectif en extension est supérieur à celui en compression, ce qui se traduit dans le diagramme $(p, q)$ de la figure $(1.4 \mathrm{c})$ par une valeur du coefficient $\mathrm{M}$ de plasticité

$$
q=M p
$$

inférieure en extension. Les chemins de contraintes effectives obtenus sont étonnamment linéaires (conduisant à une valeur du coefficient $A$ constante lors de l'écrasement).

La simulation d'essais non-drainés sur d'autres argiles ne conduit pas à ce résultat.

Les figures $(1.5 \mathrm{a}, \mathrm{b}, \mathrm{c})$ et $(1.6 \mathrm{a}, \mathrm{b}, \mathrm{c})$ correspondent à des simulations théoriques d'essais cycliques sur le même matériau argileux : la loi utilisée "Linol N.S.A. " permet en effet de simuler des chargements cycliques (Darve [7]). Sur ces figures le chargement monotone de la figure (1.4 a, b, c) a été reproduit: le chargement cyclique crée une augmentation de la pression interstitielle : l'excédent axial est beaucoup plus faible que dans l'essai monotone et les chemins de contrainte effective (fig. $1.5 \mathrm{c}$ et $1.6 \mathrm{c}$ ) rejoignent la surface limite. Des chemins comparables ont été obtenus par Sangrey [27].

\subsection{Conclusion}

La comparaison entre essais drainés et non-drainés a été effectuée sur des matériaux sableux et argileux avec une loi incrémentale non-linéaire (Loi Linol N.S.). II est possible avec ce type de loi de prévoir la pression interstitielle qui se développe lorsque les conditions d'essais imposent le non-drainage de l'échantillon. Enfin cette loi permet de simuler des cycles non drainés.

\section{Calcul de la pression interstitielle dans les ouvrages, en condition saturée non drainée}

Pour le numéricien, "ouvrage " est synonyme de champs de contraintes et de déformations hétérogènes, par opposition aux champs homogènes, censés exister uniquement dans les essais triaxiaux "correctement " antifrettés (ce qui doit être rare I).

\subsection{Les équations de base régissant la physique du phénomène}

Le développement des pressions interstitielles dans un solide poreux saturé peu perméable vis-à-vis de la vitesse des sollicitations qui lui sont appliquées, est do au couplage de volume qui existe entre le squelette et le fluide. A ce titre, ce sont les propriétés mécaniques intergranulaires du squelette qui interviennent, et non pas de quelconques «caractéristiques mécaniques non drainées équivalentes "; d'ailleurs ces caractéristiques équivalentes ne pouvent être déterminées que dans des cas très particuliers.

\subsubsection{Les inconnues (cf. fig. 2.1)}

Soit $(\Omega)$ le domaine occupé par le solide et le fluide interstitiel, de frontière commune $(\Gamma)$. Nous supposerons le couplage potentiel limité à la partie $(\omega)$ de $(\Omega)$ - physiquement, $(\omega)$ représente une zone au sein de laquelle la perméabilité serait très faible, tandis que celle de $(\Omega-\omega)$ serait très forte vis-à-vis de la vitesse de sollicitation.

Pour chaque incrément linéaire de sollicitation, les 


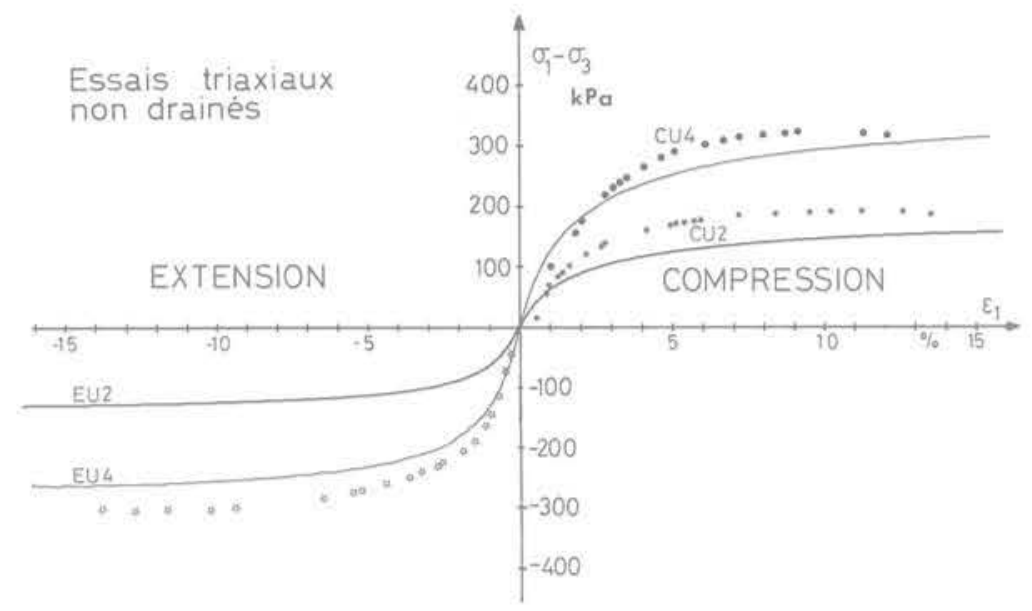

a

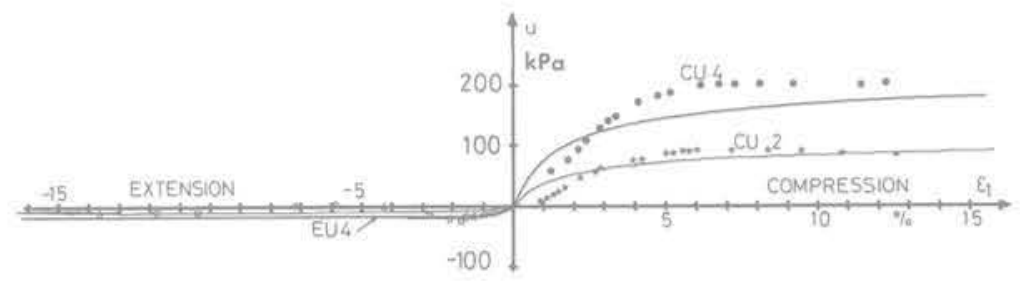

b

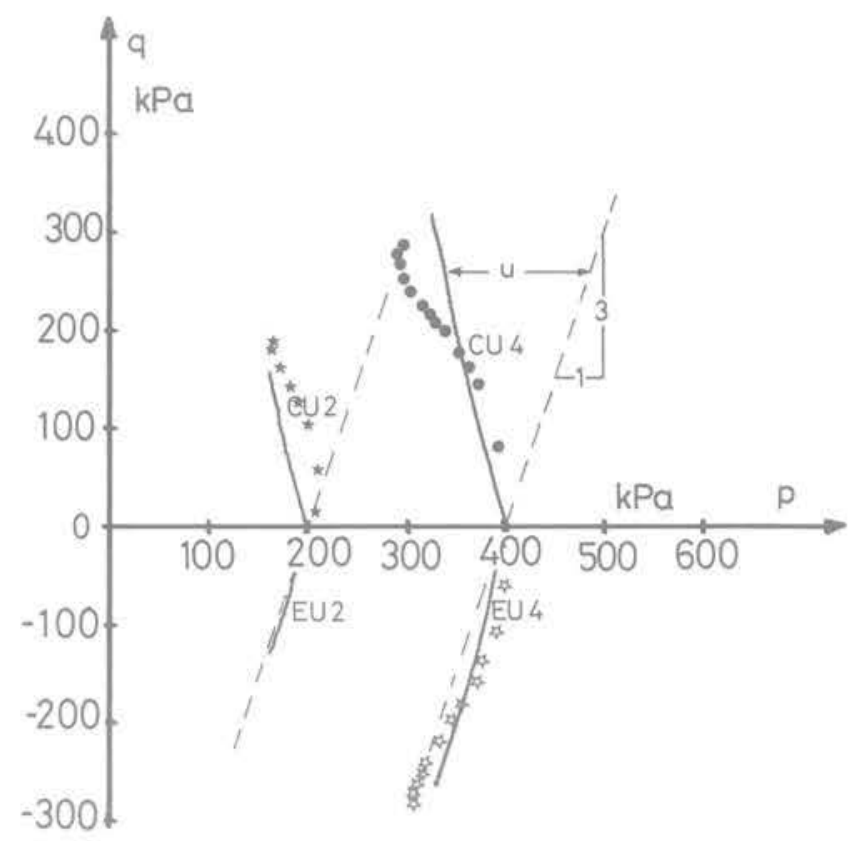

C

Fig. 1-4 Essais non-drainés sur l'argile de La Roche Chalais (points expérimentaux et courbes calculées)

inconnues en chaque point de $(\Omega)$ sont :

- le vecteur incrément de déplacement

$$
U\left(u_{i} ; i=1,2,3\right)
$$

- et le tenseur incrément de déformation qui en découle

$$
\varepsilon_{i j}(i, j=1,2,3)
$$

- le tenseur incrément de contraintes intergranulaires

$$
\sigma_{i j}(i, j=1,2,3)
$$

- l'incrément de pression interstitielle $\mathrm{p}$

Ces inconnues sont reliées entre elles par deux groupes d'équations

- les équations classiques de la mécanique des milieux continus d'une part,

- et les équations de couplage d'autre part. 

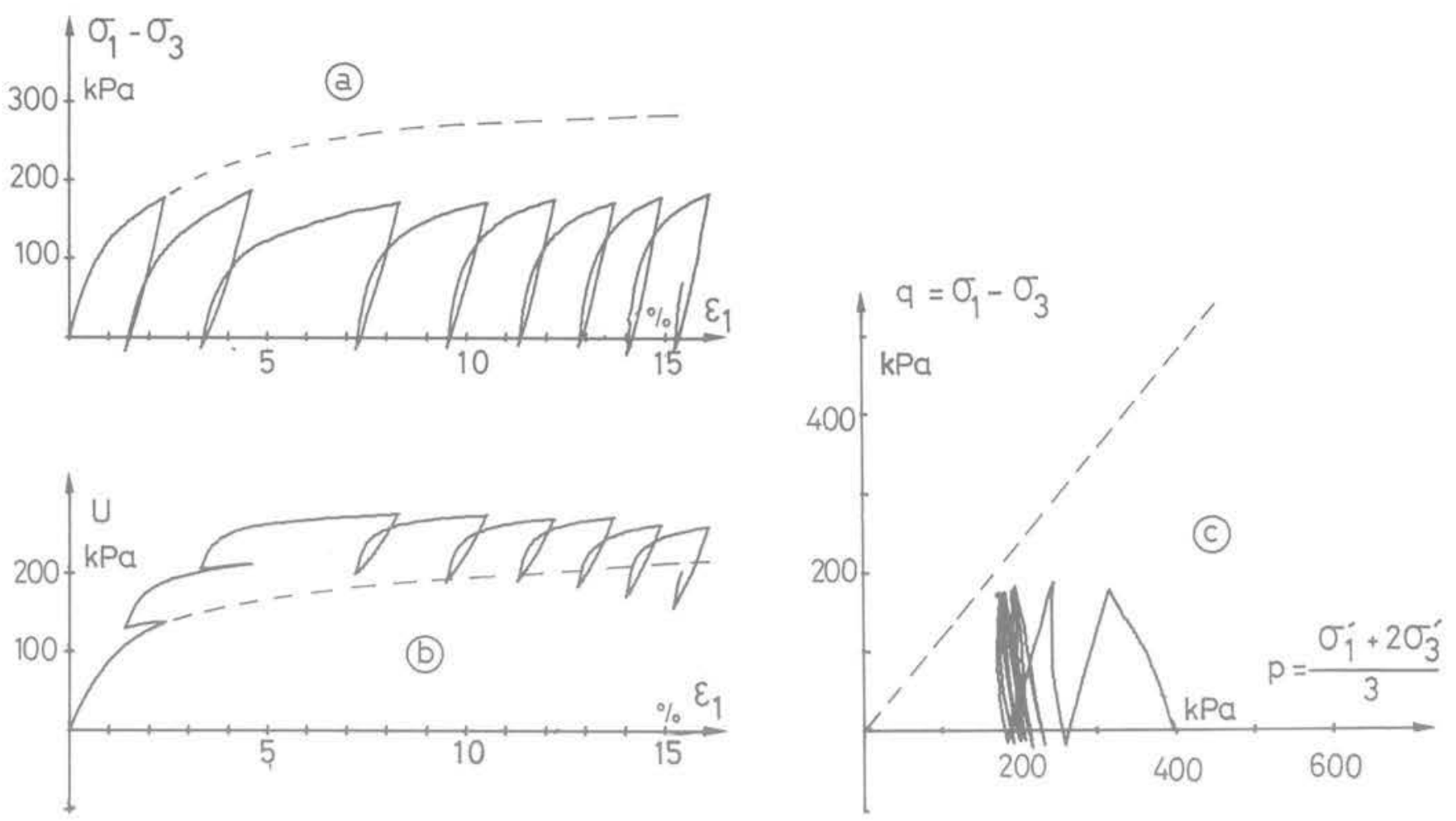

Fig. 1-5 Simulation de sollicitations cycliques sur I'argile de La Roche Chalais
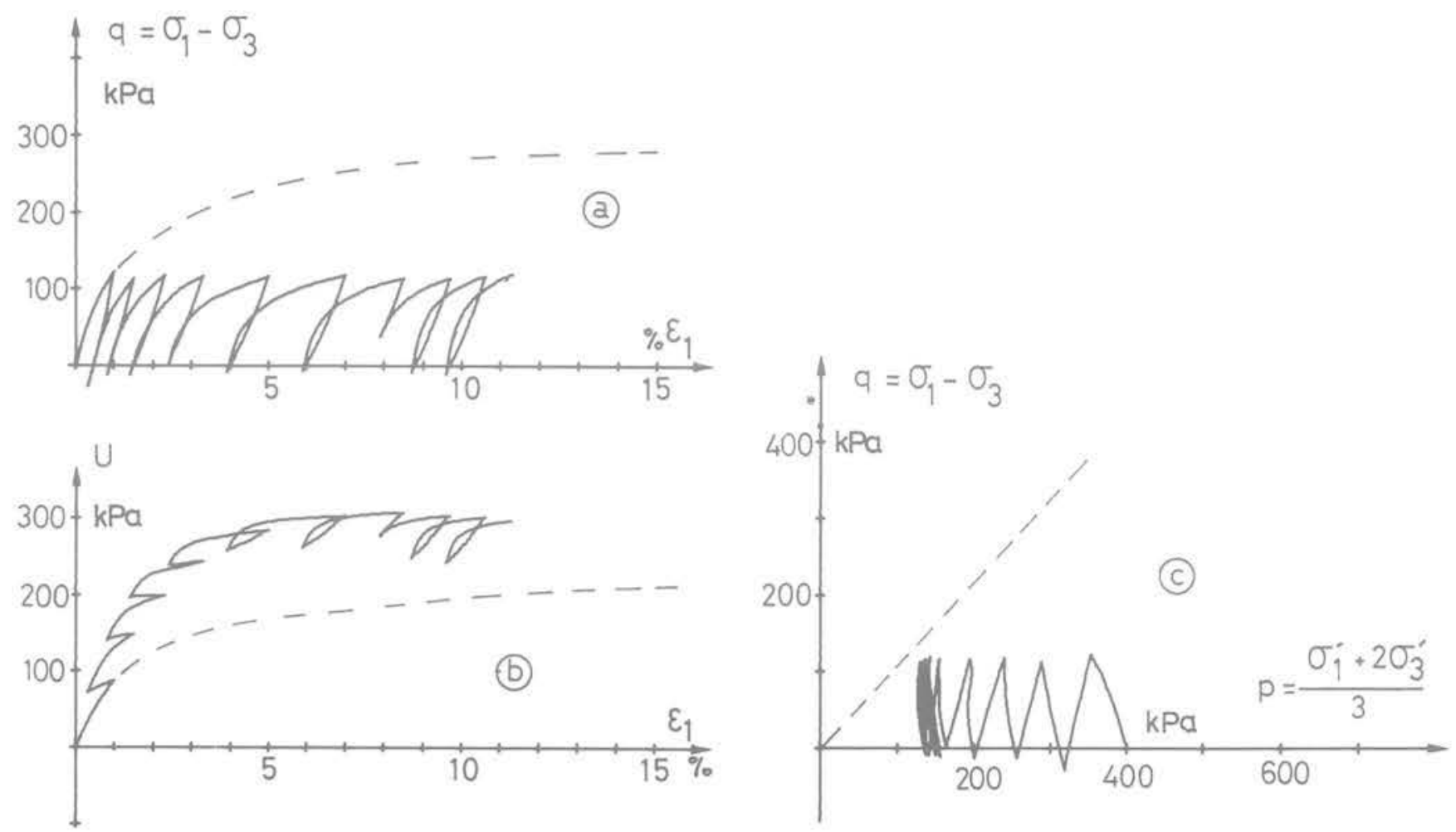

Fig. 1-6 Simulation de sollicitations cycliques sur I'argile de La Roche Chalais 


$$
\int_{\omega} \varepsilon_{i \mathrm{i}} \mathrm{dv}=0
$$
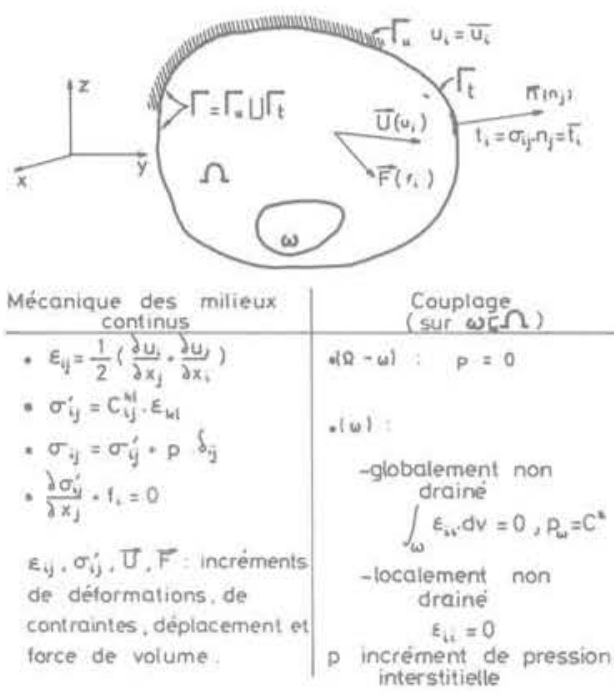

Fig. 2-1 Equations de base des problèmes de couplage en mécanique des solides saturés non-drainés

2.1.2 Les équations de la mécanique des milieux continus

a) Equations ponctuelles

En chaque point de $(\Omega)$, on peut écrire :

- la relation entre incrément de déformation et incrément de déplacement (hypothèse des petites déformations)

$$
\varepsilon_{i j}=\frac{1}{2}\left(\frac{\partial u}{\partial x_{j}}+\frac{\partial u_{i}}{\partial x_{i}}\right)
$$

- la loi rhéologique reliant les incréments de contrainte intergranulaire aux incréments de déformation

$\sigma_{i j}=C_{i j}^{k k^{\prime}} \varepsilon_{k l}$

$$
\sigma_{i j}=\sigma_{i j}^{\prime}+p \delta_{i j}
$$

- les équations de l'équilibre en termes de contraintes intergranulaires

$\frac{\partial \Sigma_{i j}^{\prime}}{\partial x_{i}}+r_{i}=u=\Sigma_{i j}^{\prime}+P \delta_{i j}$

où $\Sigma$ représente les contraintes intergranulaires (et non les

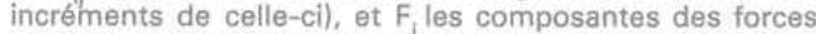
de volume (et non les incréments de celles-ci). On notera que les équations d'équilibre peuvent aussi s'écrire en termes d'incréments (par soustraction des équations (3) relatives aux incréments successifs), soit :

$\frac{\partial \sigma_{i j}^{\prime}}{\partial x_{j}}+f_{i}=0$

(f, : composantes des incréments de force de volume)

- les relations entre contraintes totales et contraintes intergranulaires (ce sont aussi des relations de couplage)

$\Sigma_{i j}=\Sigma_{i j}+P \delta_{i j}$

où $\Sigma_{\text {i }}$ représente les contraintes totales et $P$ la pression interstitielle, tandis que $\delta_{i j}$ est le symbole de Kroenecker.

L'équation précédente peut aussi s'écrire en termes d'incréments, soit :

$\sigma_{i j}=\sigma_{i j}^{\prime}+p \delta_{i j}$

\section{b) Conditions aux limites}

La frontière $(\Gamma)$ supporte

- des conditions aux limites en incréments de déplacements sur sa partie $\left(\Gamma_{u}\right)$ :

$u_{i}=u_{i}$ donné

- des conditions aux limites en contraintes sur sa partie $\left(\Gamma_{a}\right)$ :

$t_{i}=\sigma_{i j} \cdot n_{i}=t_{i}$ donné

$n_{i}(j=1,2,3)$ étant les composantes de la normale à $(\Gamma)$.

\subsubsection{Les équations de couplage}

Nous nous limitons à l'étude de sollicitations "en non-drainé " et en conséquence, nous n'abordons pas ici l'étude de la dissipation des pressions interstitielles. Mais cette phase "non-drainée » peut être considérée comme phase initiale de la consolidation. Etant donné les hypothèses précédentes, le couplage se traduit par:

- sui $(\Omega-\omega)$ : - équation ponctuelle -

$p=0$

- sur $(\omega)$

- dans le cas localement non-drainé ; équation ponctuelle :

$\varepsilon_{\mathrm{ii}}=0$

- dans le cas globalement non-drainé ; équation scalaire :

$\int_{\omega} \varepsilon_{i i} d v=0$

$\mathrm{p}=\mathrm{Cte} / \omega$

Le cas localement non-drainé se rencontre dans tous les massifs argileux, tandis que le cas globalement non-drainé est plutôt spécifique de zones perméables prisonnières de zones voisines peu perméables.

\subsection{Recensement bibliographique}

Nous examinons ici les procédés utilisés par divers auteurs pour réaliser des calculs en non drainé.

\subsubsection{Existence et obtention de la solution numérique}

L'accès à la solution numérique rigoureuse du problème non drainé se heurte à de grandes difficultés puisque le matériau est globalement incompressible. L'existence de la solution repose sur le principe variationnel très général de Hellinger-Reissner, ainsi que l'a montré Herrmann [14]. Ces travaux ont été poursuivis depuis, en éléments finis par Mikio Shoji et al [20] ainsi que par Takashi Matsumoto [29]. Mais signalons que Christian [5] obtenait dès 1968 des solutions en non drainé et en consolidation. avec une loi du sol élastique puis élasto-plastique, par la méthode des "lumped parameters ». On peut considérer que tous les auteurs cités dans ce paragraphe ont obtenu des solutions rigoureuses en ce sens que toutes les équations de leur problème aux limites sont vérifiées et qu'ils travaillent en contraintes effectives et caractéristiques mécaniques intergranulaires.

Cependant, la majorité des publications sur le sujet traite de solutions approchées, obtenues en contraintes totales avec "caractéristiques mécaniques non-drainées équivalentes ».

\subsubsection{Solutions approchées classiques}

Ces solutions sont en fait des évaluations empiriques plutôt que des calculs, puisque toutes les équations du paragraphe 2.1 ne sont pas satisfaites. Dans tous les cas, la pression interstitielle est déduite du champ des contraintes totales calculées avec des lois rhéologiques classiques. D'Appolonia et al [6] et Pilot [25] citent essentiellement quatre méthodes :

$(\Delta \mathrm{p}$ représente l'accroissement local de la pression interstitielle dans la suite). 
a $\quad \Delta p=\Delta \sigma_{\mathrm{v}}$

avec $\Delta \sigma_{\mathrm{v}}$ accroissement de contrainte verticale totale due à l'ouvrage.

$$
\Delta \mathrm{p}=\Delta \sigma_{\mathrm{oct}}=\frac{1}{3}\left(\Delta \sigma_{1}+\Delta \sigma_{2}+\Delta \sigma_{3}\right)
$$

avec $\Delta \sigma_{1}, \Delta \sigma_{2}, \Delta \sigma_{3}$ accroissements des contraintes principales et $\Delta \sigma_{\text {oct }}$ accroissement de contrainte moyenne

c $\quad \Delta \mathrm{p}=\Delta \sigma_{3}+\mathrm{A}\left(\Delta \sigma_{1}-\Delta \sigma_{3}\right)$

pour les problèmes de déformation plane :

$\Delta \sigma_{1}$ et $\Delta \sigma_{3}$ ont la même définition que précédemment ; $A$ est un des paramètres de Skempton [28], à déterminer expérimentalement.

d $\quad \Delta p=\Delta \sigma_{\text {oct }}+\alpha \cdot \Delta \tau_{\text {oct }}$

avec $\Delta \sigma_{\text {oct }}$ défini comme précédemment, $\Delta \tau_{\text {oct }}$ module du déviateur des contraintes totales, et $\alpha$ coefficient défini expérimentalement. C'est la formulation due à Henkel [13].

Z. Moh et al [22] repris par Pilot [25] présentent une comparaison du calcul selon les quatre méthodes avec l'expérience, les contraintes totales du calcul ayant été vraisemblablement obtenues en élasticité linéaire, pour la fondation (en argile molle) du remblai de Rangsit ( $3 \mathrm{~m}$ de hauteur) en Thaïlande. L'évolution de la pression interstitielle en cours de construction, montre que la méthode (a) surestime la pression interstitielle d'environ $20 \%$, tandis que les autres méthodes l'estiment correctement ou à moins de $20 \%$ près par défaut. Une évaluation par défaut est évidemment très gênante dans la mesure où elle illusionne le projeteur sur la sécurité de l'ouvrage I En ce qui concerne la fin de construction, les comparaisons mesures-calculs indiquent que les quatre méthodes surestiment les pressions interstitielles de 0 à $100 \%$, selon les profondeurs, ce qui est assez logique puisqu'un certain drainage a do avoir lieu.

D'Appolonia [6] fait le même type de comparaisons avec plusieurs hypothèses rhéologiques pour apprécier l'accroissement des contraintes totales dues à un remblai ; il s'agit ici d'une fondation constituée d'argile de Boston. normalement consolidée ou légèrement surconsolidée, enserrée entre deux couches drainantes, sur laquelle a été construit un remblai autoroutier de $13 \mathrm{~m}$ de hauteur (région Nord de Boston). En ce qui concerne les contraintes totales, on constate une divergence importante, selon la loi rhéologique utilisée dans le calcul par éléments finis : en particulier, le calcul réalisé en milieu élastique homogène sous-estime largement la variation de contrainte moyenne due au remblai à partir d'une profondeur égale à $1 / 3$ de la largeur du remblai. D'Appolonia précise que la solution considérée comme la plus correcte en contraintes totales fut obtenue avec une loi élasto-plastique à angle de frottement nul (critère de Von Mises), telle que $E_{u} / C_{u}=1200\left(E_{u}\right.$ : module d'Young non drainé équivalent ; $C_{u}$ : cohésion non drainée). Le passage de $\Delta \sigma_{\text {oct }}$ à $\Delta u$ (surpression interstitielle) permet à d'Appolonia de conclure qu'en moyenne, l'évaluation empirique $\Delta u=\Delta \sigma_{\text {oct }}$ est la plus correcte, du moins loin des couches drainantes. Par ailleurs, toutes les évaluations de $\Delta u$ donnent - dans ce cas - des valeurs supérieures à celles qui sont mesurées, ce qui va dans le sens de la sécurité.

L'article de Moh donne un résultat contraire I Les conclusions sont délicates...

\subsection{Notre méthode de calcul}

Notre méthode n'est pas originale puisqu'elle était déjà utilisée, à quelques variantes près par Christian [5], mais elle est applicable à un sol possédant une loi rhéologique quelconque, décrite de manière incrémentale.
Nous opérons en contraintes effectives et utilisons exclusivement les caractéristiques mécaniques intergranulaires du sol, ainsi que la physique le suggère. II est à noter que, dans ces conditions, le squelette n'est nullement incompressible, mais que chaque élément de volume, du moins dans le cas localement non drainé, suit un chemin en déformation à volume constant.

\subsubsection{Principe du calcul}

Chaque incrément linéaire de sollicitation est traité en 4 étapes utilisant chacune les paramètres intergranulaires du sol (cf. fig. 2.2, pour le cas localement non-drainé).

\section{a) Etape 1}

On résoud le problème correspondant à l'incrément de sollicitation comme si le matériau était parfaitement drainé. On en déduit les champs incrémentaux suivants sur $(\Omega)$ :

champ de déplacements $(\vec{U})_{1}$ champ de déformations $\left(\varepsilon_{i j}\right)$, champ de contraintes $\left(\sigma_{i j}\right)_{1}$

La champ $\left(\varepsilon_{\mathrm{ij}}\right)$, conduit aux incréments de variations de volume ponctuelles $\left(\varepsilon_{\mathrm{kk}}\right)_{1}=\left(\varepsilon_{v}\right)_{1}$

b) Etape 2

On détermine les fonctionnelles suivantes :

$$
\begin{aligned}
& u_{i}(P) & =F[p(Q)] \\
\text { et } & \varepsilon_{i j}(P) & =G[p(Q)]
\end{aligned}
$$

où les $u_{i}$ et les $\varepsilon_{i j}$ sont respectivement les composantes des incréments de déplacements et celles du tenseur des incréments de déformations, tandis que $\mathrm{P}$ est le point courant de $(\Omega)$ et $Q$ le point courant de $(\omega)$ et que $p(Q)$ représente un champ de contrainte isotrope sur $(\omega)$.

Ces fonctionnelles sont obtenues avec des conditions aux limites spéciales, à savoir:

- conditions aux limites en déplacements :

$u_{i}=0$ sur $\left(\Gamma_{u}\right)$

- conditions aux limites en contraintes:

$\sigma_{i j} \cdot n_{j}=0$ sur $\left(\Gamma_{\sigma}\right)$

On peut déduire de (18) la fonctionnelle représentant les incréments de variations de volume ponctuelles:

$\varepsilon_{v}(P)=H[p(Q)]$

\section{c) Etape 3}

Il s'agit d'inverser l'équation fonctionnelle (22) de manière à trouver un champ d'incréments de contrainte isotrope (w) qui annule les incréments de variations de volume de l'étape 1 ; le champ $\left(\varepsilon_{k k}\right)$, de l'étape 1 est une quantité connue dans cette phase.

Dans le cas localement non drainé, on doit trouver la fonction $p(Q)-Q \in(\omega)^{\prime}-$ telle que :

$H[p(Q)]=-\left(\varepsilon_{\mathrm{kk}}\right)_{1}=-\left(\varepsilon_{\mathrm{v}}\right)_{1}$

- et dans le cas globalement non drainé, on cherche l'incrément de pression isotrope $p$, constante sur $\omega$, telle que :

$H[p]=-\frac{\int_{\omega}\left(\varepsilon_{k k}\right)_{1} d V}{\int_{\omega} d V}$

Connaissant la fonction $p(Q)$ dans le cas localement nondrainé, et p dans le cas globalement non-drainé, on calcule sur $(\Omega)$ les champs incrémentaux: 


\begin{tabular}{|c|c|c|}
\hline ETAPE & PROBLEME & RESULTAT \\
\hline $\begin{array}{c}1 \\
\text { PROBLEME } \\
\text { DRAINE }\end{array}$ & & $\begin{array}{l}\left(u_{i}\right)_{1}^{\text {CHAMPS }} \\
\left(\varepsilon_{i j}\right)_{1} \Rightarrow\left(\varepsilon_{v}\right)_{1} \\
\left(\sigma_{i j}\right)_{1}\end{array}$ \\
\hline $\begin{array}{l}2 \\
\text { FONCTIONNELE } \\
\text { D'INFUUENCE }\end{array}$ & $\therefore u_{i}=0$ & $\begin{aligned} \text { FONCTIONNELLES } & \\
u_{i}(P)= & =[p(Q)] \\
E_{j}(P) & =\mathscr{y}[p(Q)] \\
& \Downarrow \\
\varepsilon(P) & =\nVdash[p(Q)]\end{aligned}$ \\
\hline \begin{tabular}{|c|} 
\\
\multicolumn{1}{|c|}{} \\
CALCUL DE \\
LA PRESSION \\
INTERSTITIELLE
\end{tabular} & $\begin{array}{c}\text { INVERSION FONCTIONNELLE } \\
H_{[}[p(Q)]=-\left(\varepsilon_{v}\right)_{1}\end{array}$ & $\begin{array}{c}\text { CHAMPS } \\
\left(u_{i}\right)_{3} \\
\left(\varepsilon_{i j}\right)_{3} \Rightarrow\left(\varepsilon_{i}\right)=-\left(\varepsilon_{i}\right) \\
\left(\sigma_{i j}\right)_{3}=p(Q) . \delta_{i j}\end{array}$ \\
\hline $\begin{array}{l}4 \\
\text { PROBLFME } \\
\text { NON DRAINE. }\end{array}$ & remarque: $\left(E_{v}\right)_{4}=0$ & $\begin{aligned} \text { CHAMPS } \\
\left(u_{i}\right)_{4}=\left(u_{i}\right)_{1}-\left(u_{i}\right)_{3} \\
\left(\varepsilon_{i j}\right)_{4}=\left(\varepsilon_{i j}\right)_{3}-\left(\varepsilon_{i j}\right)_{3} \\
\left(\sigma_{i j}\right)_{4}=\left(\sigma_{i j}\right)_{4}-\left(\sigma_{i j}\right)_{3} \\
\left(\sigma_{i,)_{4}}=\left(\sigma_{i i}\right)_{1}\right. \\
(p)_{4}=\left(\sigma_{i j}\right)_{3}\end{aligned}$ \\
\hline
\end{tabular}

Fig. 2-2 Principe de résolution en non-drainé incrémental

- de déplacements (U) 3 grâce à (17)

- de déformations $\left(\varepsilon_{i j}\right)_{3}$ grâce à (18) ou à (1)

- et de contraintes $\left(\sigma_{i j}\right)_{3}$ grâce à (2)

Notons que $\left(\sigma_{i i}\right)_{3}$ représente sur $(\omega)$, le champ de pression isotrope cherché.

\section{d) Etape 4}

On superpose les champs indicés 1 et 3 . La solution du problème incrémental non-drainé est donc :

$(\vec{U})=\langle\vec{U})_{1}-(\vec{U})_{3}$ en déplacements

$\left(\varepsilon_{i j}\right)=\left(\varepsilon_{i j}\right)_{1}-\left(\varepsilon_{i j}\right)_{3}$ en déformations ; $\varepsilon_{\mathrm{kk}}=0 \operatorname{sur}(\omega)$

$\left(\sigma_{i}\right)=\left(\sigma_{i}\right)_{1}-\left(\sigma_{i}\right)_{3}$ en contraintes intergranulaires

$p=\left(\sigma_{i j}\right)_{3} \operatorname{sur}(\omega)$

$p=0$ sur $(\Omega-\omega)$

\subsubsection{Technologie de la méthode}

Le problème est résolu numériquement par la méthode des éléments finis en déplacements, à laquelle on joint un algorithme spécial réalisant les étapes 2, 3 et 4 ci-dessus (cf. fig. 2.3).

Le point délicat réside évidemment dans la détermination des fonctionnelles de l'étape 2, puisque le reste du calcul est parfaitement classique. Dans le problème incrémental, ces fonctionnelles sont linéarisées puisque les propriétés mécaniques sont constantes sur un incrément. Tout revient donc à déterminer le tableau des coefficients d'influence d'un élément sur l'autre par une méthode de contraintes initiales nous conduisant à un champ de contraintes isotropes sur $(\omega)$. Pour les géométries où $4 \mathrm{com-}$ posantes du tenseur contrainte sont non nulles (axisymétrie, déformation plane, contraintes planes), il y a 4 sys-

\begin{tabular}{|c|c|c|}
\hline ETAPE & PROBLEME & RESULTAT \\
\hline $\begin{array}{c}1 \\
\text { PROBLEME } \\
\text { DRAINE }\end{array}$ & intions & $\begin{array}{l}\text { CHAMPS } \\
\left(u_{i m}\right), \\
\left(\varepsilon_{i j \lambda}\right), \Rightarrow\left(\varepsilon_{i \lambda}\right), \\
\left(\sigma_{i j \lambda}\right), \\
i, j=1,2,3\end{array}$ \\
\hline \begin{tabular}{|c|}
2 \\
COEFFICIENTS \\
D'INFLUENCE
\end{tabular} & $=0 \operatorname{son} \quad-T_{u} u_{i}=0$ & $\begin{array}{l}\text { COEFFICIENTS } \\
F_{i m}^{\lambda}, G_{i j}^{\mu \lambda}, H_{\mu}^{\lambda} \\
u_{i m}=F_{i m}^{\lambda} \cdot p_{\lambda} \\
\varepsilon_{i j}^{\mu}=G_{i j}^{\mu \lambda} \cdot p_{\lambda} \\
\varepsilon_{v \mu}=H_{\mu}^{\lambda} \cdot p_{\lambda}\end{array}$ \\
\hline \begin{tabular}{|c|}
\multicolumn{2}{|c|}{3} \\
CALCUL DE \\
LA PRESSION \\
INTERSTITELLE
\end{tabular} & $\begin{array}{r}\text { RESOLUTION SYSTEME } \\
\text { ALGEBRIQUE LINEAIRE } \\
H_{\lambda}^{\mu} \cdot P_{\mu}=-\left(\varepsilon_{v \lambda}\right)_{1}\end{array}$ & $\begin{array}{l}\text { CHAMPS } \\
\left(u_{i m}\right)_{3} \\
\left(E_{i j \lambda}\right)_{3} \\
\left(\sigma_{i j \lambda}\right)_{3}=p_{\lambda} \cdot \delta_{i j}\end{array}$ \\
\hline $\begin{array}{c}4 \\
\text { PROBLEME } \\
\text { NON DRAINE }\end{array}$ & remarque : $\left(\varepsilon_{v} \xi_{i}=0\right.$ & $\begin{aligned} \text { CHAMPS } \\
\left(u_{i m_{4}}\right)_{4}=\left(u_{i m}\right)^{\lambda}-\left(u_{m}\right) \\
\left(\varepsilon_{i j}^{\lambda}\right)_{4}=\left(\varepsilon_{i j}^{\lambda}\right)_{1}-\left(\varepsilon_{i j}^{\lambda}\right) \\
\left(\sigma_{i j}^{\lambda}\right)_{4}=\left(\sigma_{i j}^{\lambda}\right)_{1}-\left(\sigma_{i j}^{\lambda}\right) \\
\left(\sigma_{i j}^{\lambda}\right)_{4}=\left(\sigma_{i j}^{\lambda}\right)_{1}\end{aligned}$ \\
\hline
\end{tabular}

Fig. 2-3 Principe de résolution en non-drainé incrémental linéarisé

tèmes linéaires à résoudre par élément. Comme tous ces systèmes linéaires sont construits à l'aide des mémes caractéristiques mécaniques (intergranulaires), il suffit de résoudre $4 . \mathrm{N}$ cas de charge supplémentaires pour un problème comportant $\mathrm{N}$ éléments. Les coefficients d'influence, sont de la forme $\mathrm{H}_{\lambda \mu}$ tels que :

$\left(\varepsilon_{v}\right)_{\lambda}=H_{\lambda \mu} \cdot(p)_{\mu}$

$\lambda$ et $\mu$ étant respectivement les indices des éléments courants du domaine $(\omega)$ et du domaine $(\Omega)$.

\subsection{Résultats obtenus}

Nous avons réalisé deux séries de calculs : la première avec loi rhéologique élastique pour un pieu, et la seconde avec loi incrémentale pour un triaxial fretté. Nous ne possédons pas encore de vérifications expérimentales de ces calculs.

\subsubsection{Pieu en sol élastique non drainé}

Le pieu est considéré comme rigide; le sol possède les caractéristiques élastiques suivantes :

$E^{\prime}=50 \mathrm{MPa}$ et $v^{\prime}=0.3$

(E' module d'Young, et $v^{\prime}$ coefficient de Poisson drainés).

Sa longueur est de $8 \mathrm{~m}$ et son diamètre vaut $30 \mathrm{~cm}$ (cf. fig. 2.4).

La figure 2-5 donne le champ des variations de volume en drainé, tandis que la figure 2-6 représente le champ de pressions interstitielles, pour un arrachement relatif W/B de $1 / 100$. On voit que la tendance à la dilatance se trouve confinée sous la pointe, tandis que la tendance à la contractance est concentrée près de la base du fût. Le développement des pressions interstitielles est en accord avec ces tendances. On peut prévoir des écoulements importants de la base du fât vers la pointe dans la période initiale de consolidation. 


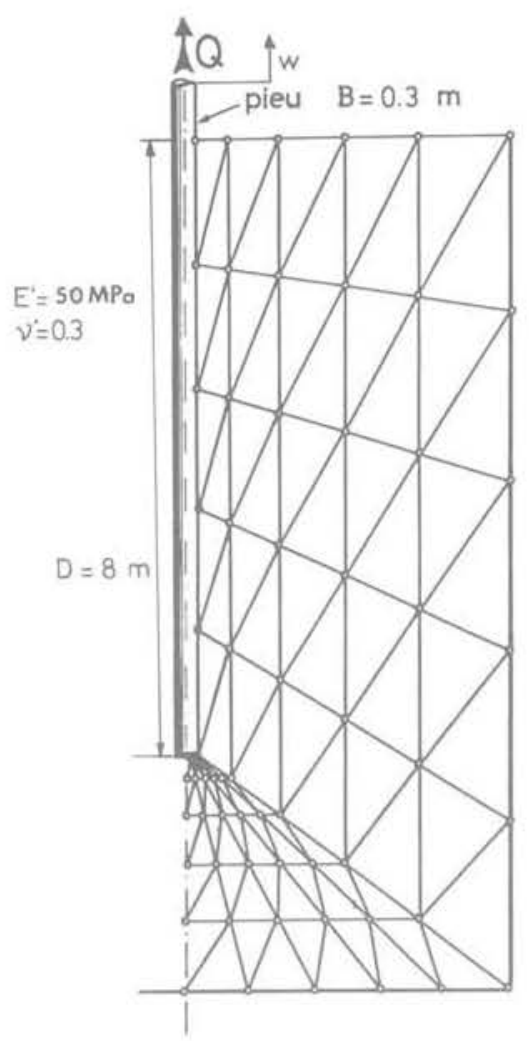

Fig. 2-4 Arrachement de pieu : maillage (sol élastique non drainé)

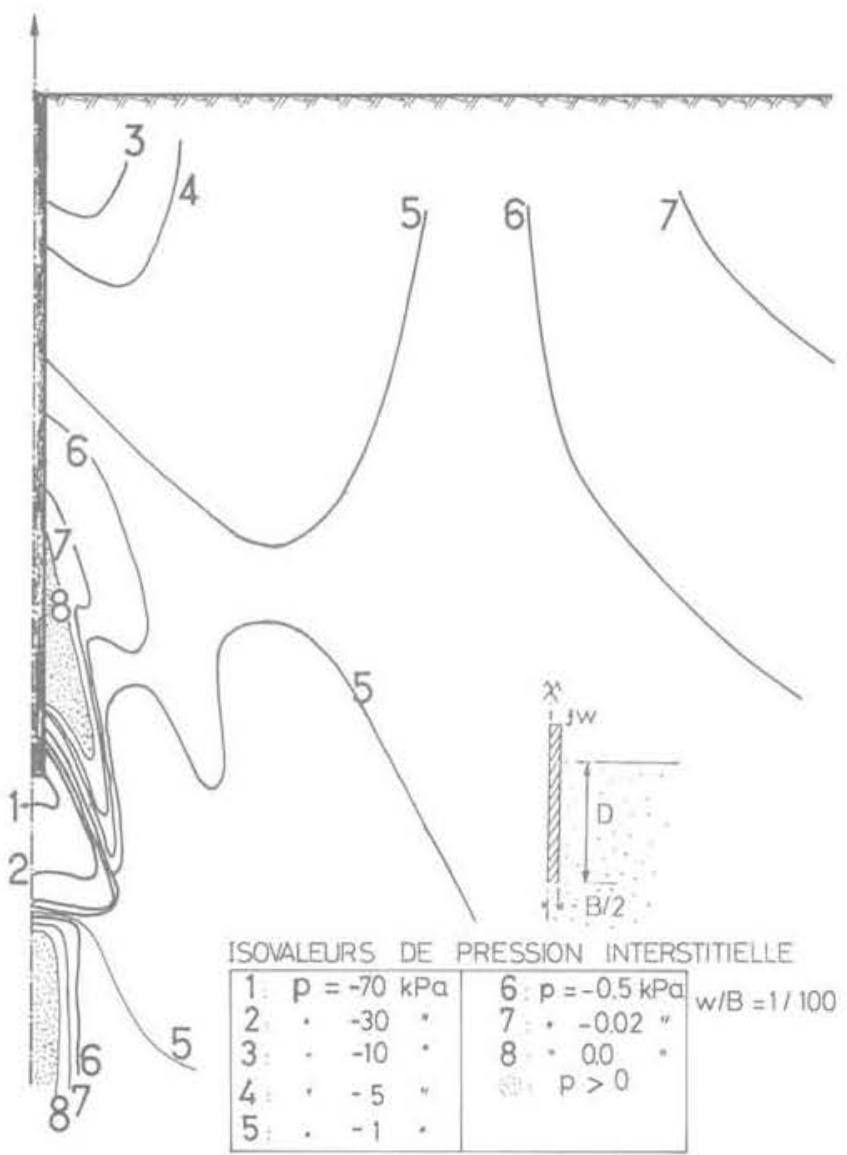

Fig. 2-6 Champ de pressions interstitielles - phase 3 sol élastique

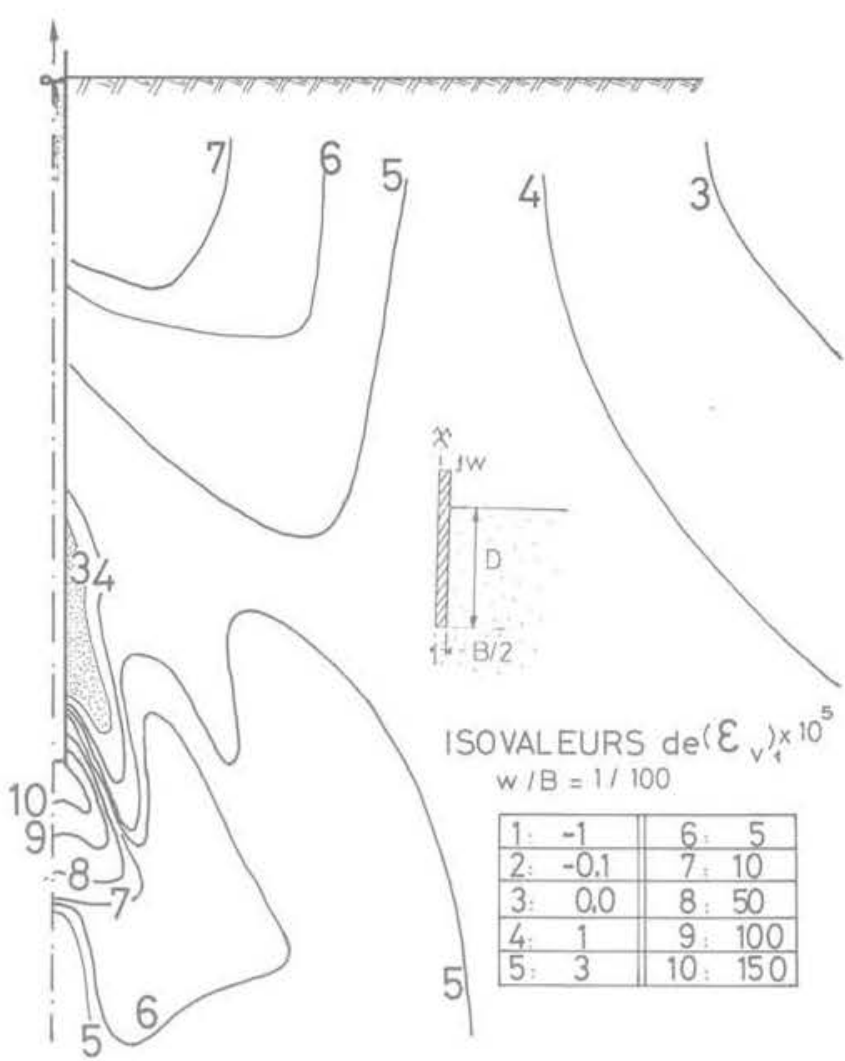

Fig. 2-5 Champ des variations de volume - phase 1 sol élastique

La figure 2-7 présente l'action sol sur fût en drainé et en non-drainé. La comparaison est particulièrement intéressante du point de vue des contraintes de cisaillement (leur résultante en non-drainé - c'est-à-dire la force d'arrachement - est neuf fois plus faible qu'en drainé). Mais ce résultat est à manier avec circonspection car en même temps les contraintes normales intergranulaires sur le fût sont fortement en traction, ce qui implique des glissements relatifs modifiant l'état des contraintes.

\subsubsection{Essai triaxial fretté non drainé}

Lors du maniement de notre loi incrémentale dans les calculs par éléments finis, se pose le problème du choix des caractéristiques mécaniques ponctuelles puisque ces caractéristiques (pseudo-module et pseudo-coefficient de Poisson) sont fonction du sens de variation des contraintes au cours de l'incrément calculé. Le nombre de possibilités est énorme ( $8^{N}$ pour $N$ éléments) et notre algorithme de choix n'est pas encore parfaitement au point. Toutefois, dans le cas de l'essai triaxial, le choix des zones tensorielles est assez facile. Les résultats de cette méthode appliquée au cas d'un pieu sont indiqués figure 2-8. Avant un incrément, on fait une hypothèse sur le sens de variation des contraintes selon chaque direction ponctuelle d'orthotropie du matériau, et on vérifie la validité de ce choix au terme du calcul; si le choix est erroné, on le modifie et on recommence.

\section{a) Essai localement non-drainé}

II est réalisé sur un matérieu pulvérulent supposé extrêmement fin. L'échantillon est un cylindre de $10 \mathrm{~cm}$ de diamètre et $10 \mathrm{~cm}$ de hauteur; il est supposé parfaitement fretté par les têtes. Le calcul est axisymétrique et est réalisé dans un plan méridien sur le 1/4 de l'échantillon.

La figure 2-9 donne les champs de pressions interstitielles pour deux valeurs de $\varepsilon_{1}$ : les fortes pressions interstitielles sont concentrées vers le centre de l'échantillon. 

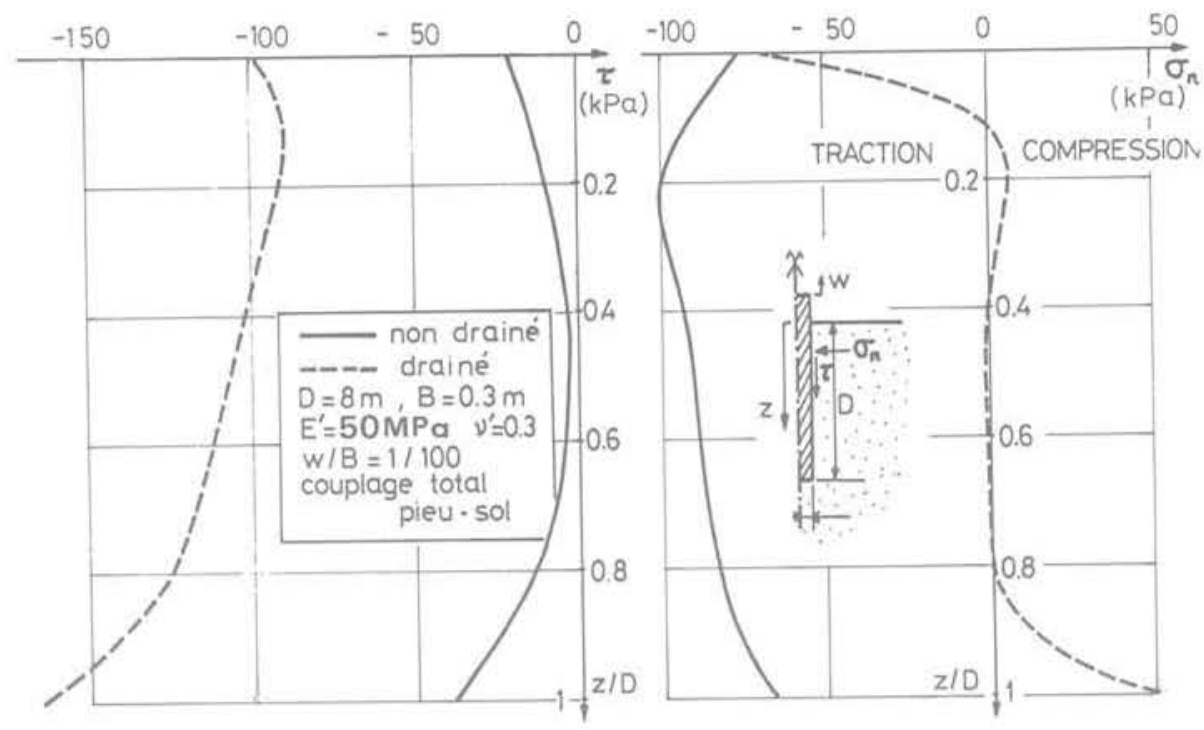

Fig. 2.7 Arrachement : Action sol - pieu : comparaison drainé - non drainé

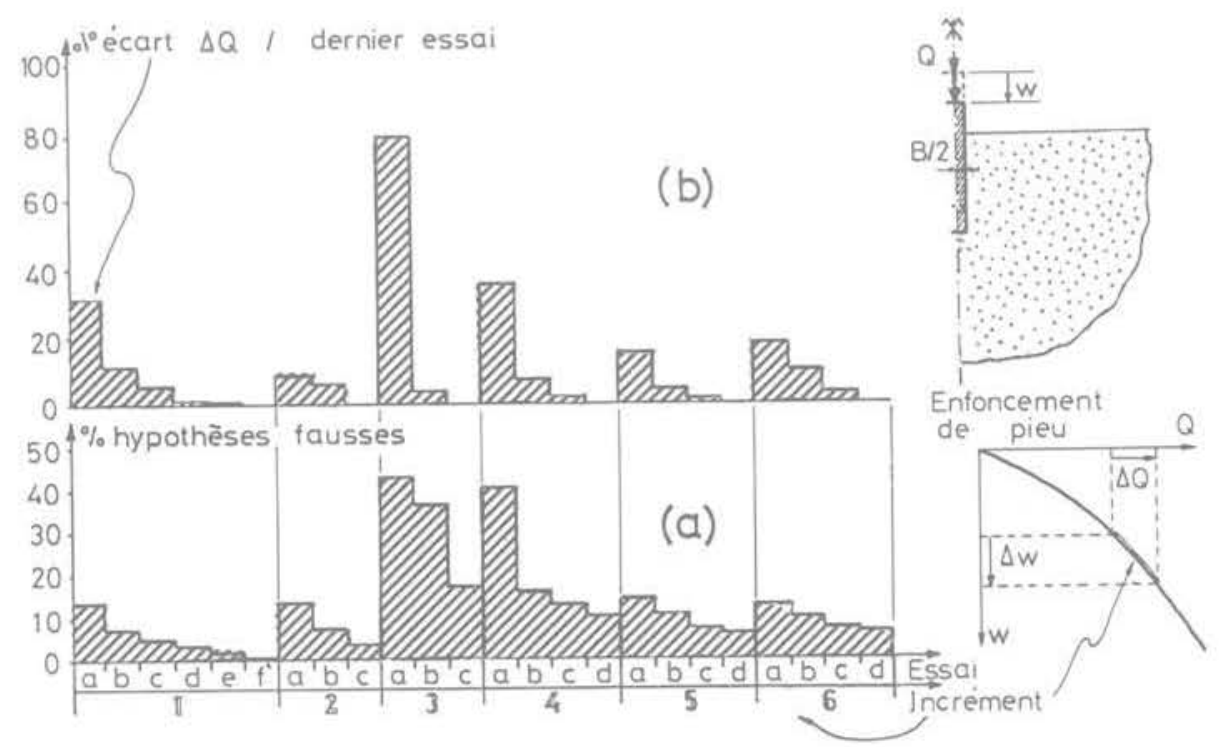

Fig. 2-8 Incidence du choix des zones tensorielles sur le résultat global

La figure 2-10 donne les isovaleurs de $\sigma_{1}^{\prime} / \sigma_{3}^{\prime}$ pour trois valeurs de $\varepsilon_{1}$. On voit que l'échantillon est extrêmement hétérogène de ce point de vue, et que le centre de l'échantillon est bien plus proche de la rupture que les zones situées près des têtes.

$\Phi^{\prime}=35^{\circ}, \sigma^{\prime}, / \sigma_{3}^{\prime}$ rupture $\# 3.8 ; \sigma_{3}=100 \mathrm{kPa}$

La figure 2-11 donne l'évolution de $u, \sigma_{1}^{\prime}, \sigma_{3}^{\prime}$ et $\sigma_{1}^{\prime} / \sigma_{3}^{\prime}$ au centre et au pied de l'échantillon, en fonction de $\varepsilon_{1}$. On voit bien encore la grande hétérogénéité de l'échantillon, le fait que les diverses zones arrivent à la rupture pour des valeurs différentes de $\varepsilon_{1}$. II est intéressant de constater que la pression interstitielle finale en pied est deux fois plus faible qu'au centre de l'échantillon, ce qui est corroboré par l'expérience, et provient probablement du fait que le frettage des têtes réduit la tendance à la contractance de l'échantillon.

II est intéressant de noter aussi que les valeurs finales de $\sigma^{\prime}$ et $\sigma_{3}^{\prime}$ (au palier) sont sensiblement les mêmes en pied et au centre, ce qui rend toute sa valeur à cet essai, même " hétérogène ». 

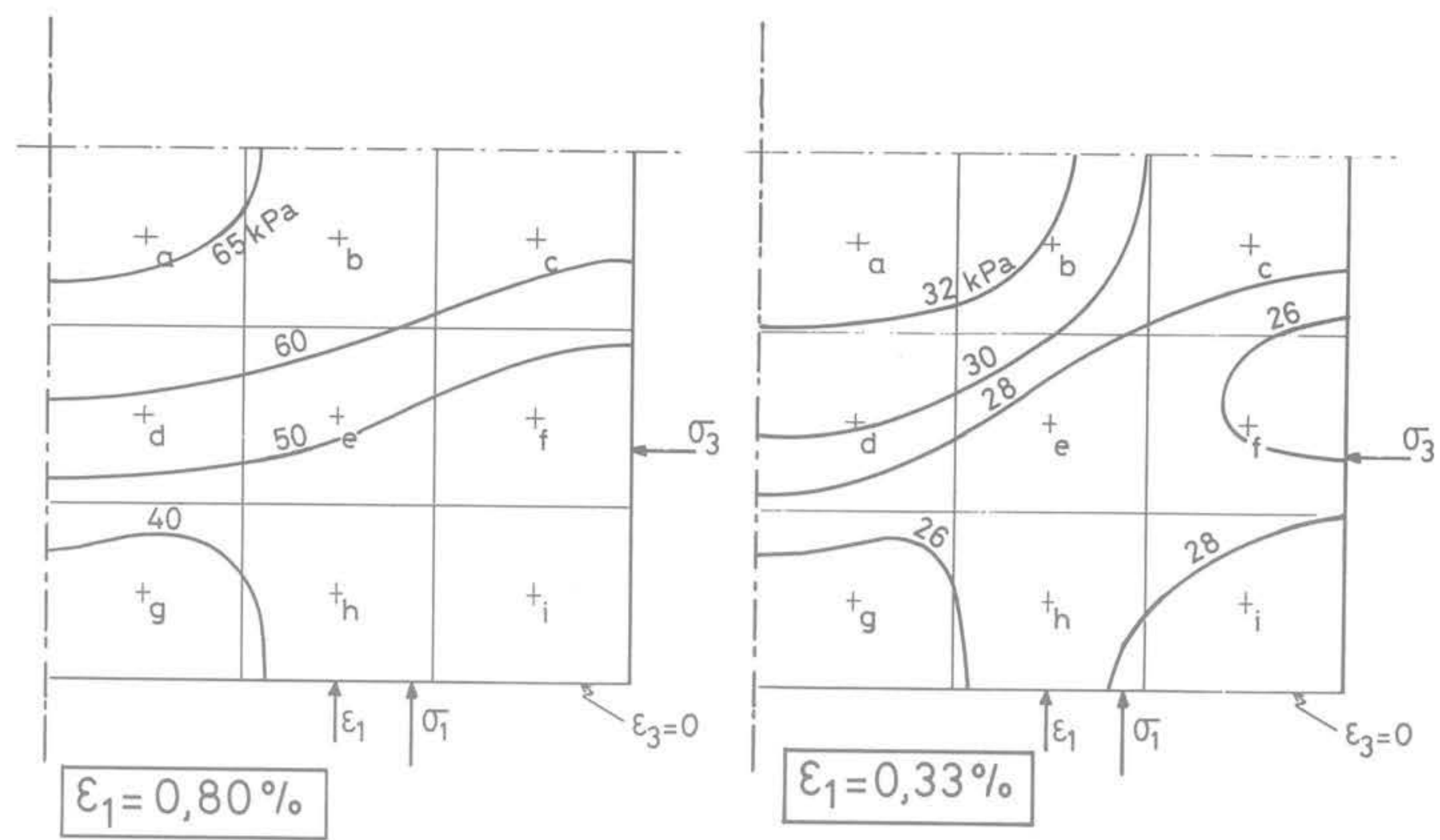

Fig. $2-9$ Isovaleurs des pressions interstitielles (localement non-drainé)
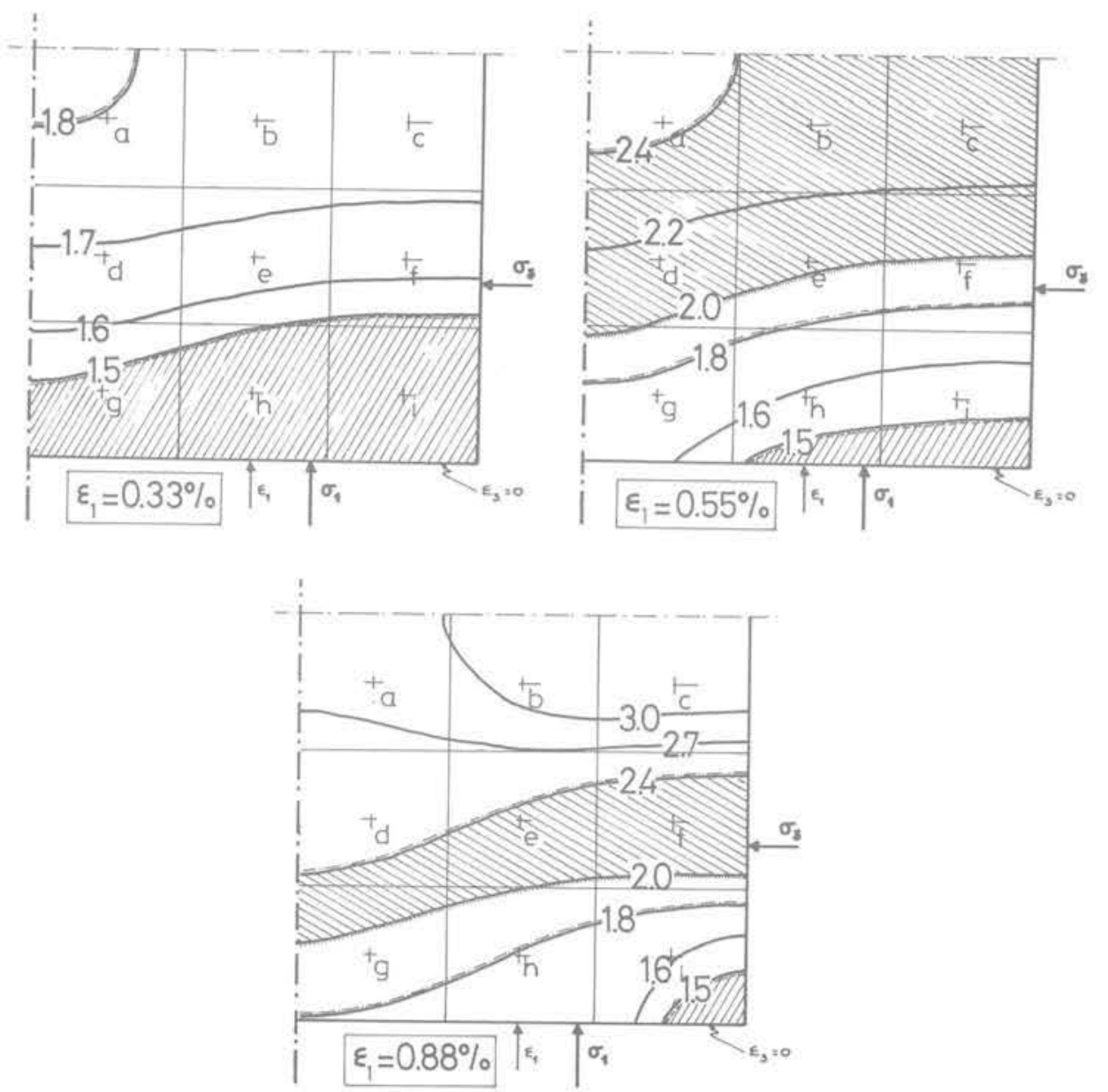

Fig. 2-10 Isovaleurs du rapport $\sigma_{1}^{\prime} / \sigma_{3}^{\prime}$ (localement non-drainé) 

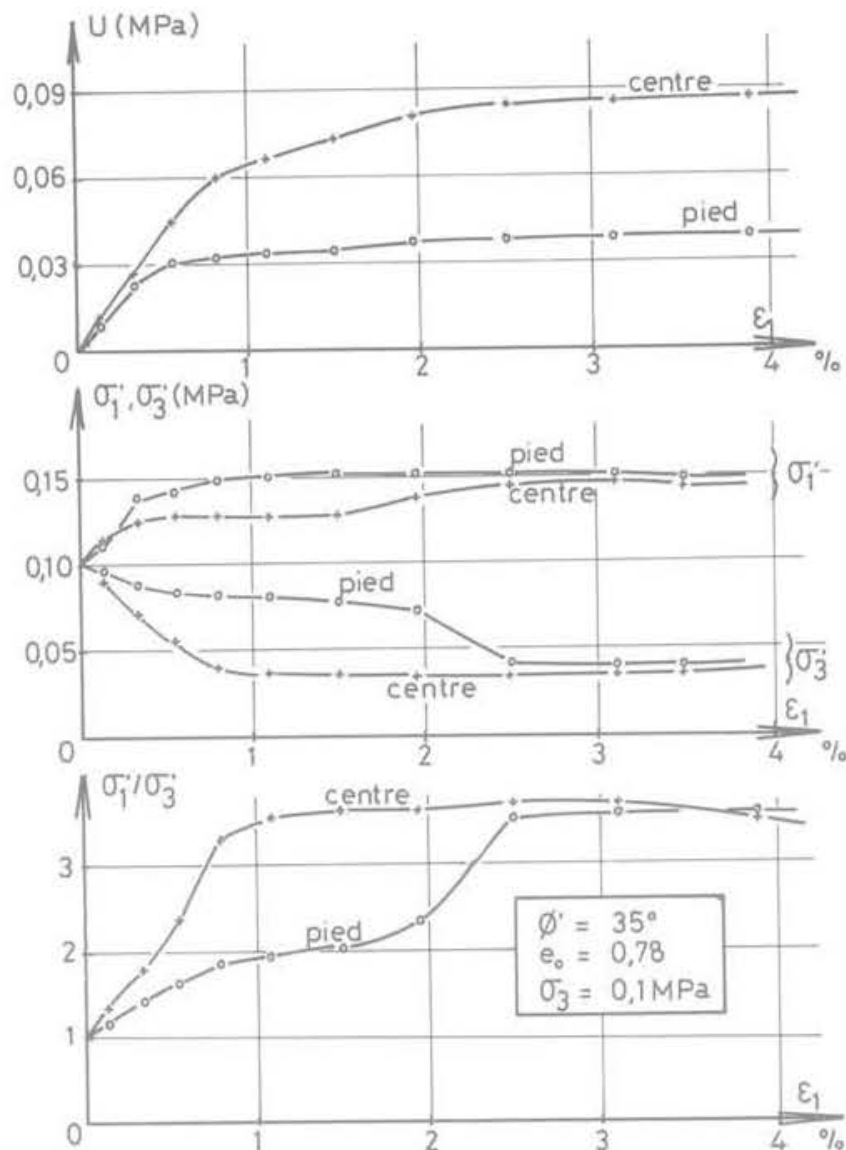

Fig. 2-11 Essai triaxial fretté localement non-drainé

\section{b) Essai globalement non-drainé}

La figure 2-12 rassemble tous les résultats intéressants de ce type de calcul, et en particulier l'évolution de la pression interstitielle dont la valeur finale est sensiblement la même qu'en localement non-drainé, mais dont la vitesse initiale de croissance est plus élevée.

On note par ailleurs que le centre de l'échantillon est beaucoup plus proche du palier de plasticité que les parties proches de la tête; c'est que les déformations près des sont considérablement gênées, tandis qu'elles ne le sont pas vers le centre, ce qui permet à l'échantillon de se dilater dans cette zone, alors qu'il se contracte ailleurs. Ceci est confirmé par le fort rapport $\sigma_{1}^{\prime} / \sigma_{3}^{\prime}$ de palier au centre (\# 5,2) correspondant à une faible densité, alors que ce rapport est de l'ordre de 2,8 près des têtes, ce qui correspond à une densité plus forte que la densité initiale.

\section{Conclusion}

Les résultats obtenus permettent d'affirmer qu'on peut actuellement procéder à des analyses assez fines de la rhéologie des sols et du comportement des ouvrages, en contraintes effectives et en utilisant les seules propriétés mécaniques intrinsèques d'un matériau: ses propriétés intergranulaires. Nous nous attachons actuellement à perfectionner la loi rhéologique utilisée dans la simulation notamment en ce qui concerne la dépendance directionnelle, ainsi que le comptage des divers algorithmes nécessaires à l'utilisation par la méthode des éléments finis (mise en œuvre d'une loi à dépendance directionnelle. interaction sol-structure et interaction sol-fluide interstitiel).
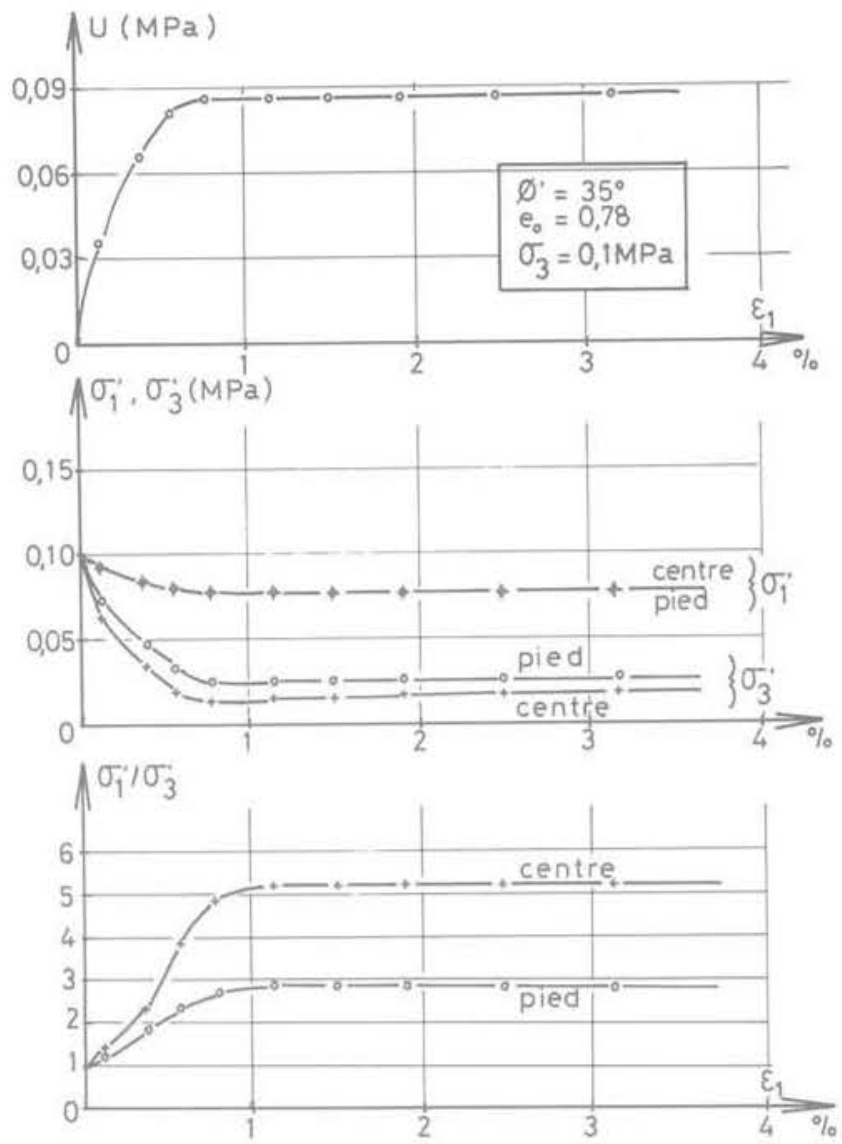

Fig. 2-12 Essai triaxial fretté globalement non-drainé

\section{Références Bibliographiques}

[1] ANQUETIL J. (1976) - "Déformation des sables sous différents états de contraintes"Thèse de Spécialité. Université de Paris VI.

[2] BOULON M., CHAMBON R., DARVE F. (1977) - "Loi rhéologique incrémentale pour les sols et application parla méthode des éléments finis". Revue Française de géotechnique, $n^{\circ} 22$, pp. 5-22, Sept. 1977.

[3] BOUTWELL G.P. (1968) - "On the yield behavior of cohesionless materials" Soil Mechanics Series $n^{\circ} 7$. Duke University. U.S.A.

[4] CHAMBON R., RENOUD-LIAS B. (1979) - "Incremental non linear stress-strain relationship for soils and integration by F.E.M."

3rd Int. Conf. on Numerical Method in Geomechanics 1, pp. 403-413, Ed. W. WITTKE.

[5] CHRISTIAN J.T. (1968) - "Undrained stress distribution by numerical methods". J. of Soil Mechanics and Foundation Engineering Proceedints of the American Society of Civil Engineers, SM 6, pp. 1333-1345, Nov., 1968.

[6] D'APPOLONIA D.J., LAMBE T.W., POULOS H.G. (1971) - "Evaluation of pore pressure beneath an enbankement". J. of Soil Mechanics and Foundation Engineering. Proceedings of the American Society of Civil Engineers, $n^{\circ}$ SM 6, pp. 881-897, June 1971.

[7] DARVE F. (1978) - "Une formulation incrémentale des lois rhéologiques. Application aux sols". Thèse d'Etat, Université de Grenoble.

[8] DARVE F., LABANIEH S., CHAMBRON R. (1976) "Incremental stress-strain relationship for cohesionless oil" Second Int. Conf. on Numerical Methods in Geomechanics 1, pp. 264-269 Ed. C. DESAI. 
[9] DARVE F., BOULON M., CHAMBON R. (1978) - "Loi rhéologique incrémentale des sols". Journal de Mécanique, 17, n०5, pp. 679-716. (1978).

[10] DARVE F., FLAVIGNY E., VUAILLAT P. (1979) "Une lopi rhéologique complète pour matériaux argileux". $7^{\circ}$ Conf. Europ. de Mécanique des Sols et Travaux de Fondation, vol. I. pp. 119-124.

[11] DUNCAN J.M., CHANG C.Y. (1970) - "Non linear analysis of stresse and strain in soils". J. of the Soil Mechanics and Foundations Division. Proc of the American Society of Civil Engineers. Vol. 96, n० SM 5, pp. 1629-1653, May 1970.

[12] FLAVIGNY E. (1978) - "Trois aspects des propriétés mécaniques des sols". Thèse de Spécialité, Université de Grenoble.

[13] HENKEL D.J. (1960) - "The shear strength of saturated remolded clays". Proc, of the research conf. on shear strength of soils. American Society of Civil Engineers, Boulder, Colorado, pp. 533-554, June 1960.

[14] HERRMAN L.R. (1965) - "Elasticity equations for incompressible and nearly incompressible materials by a variationnal theorem". Publication of the American Institute of Aéronautics and Astronautics, AIAA Journal, Vol. 3, $n^{\circ}$ 10.

[15] KERISEL J. (1977) - Séance de clôture IX Int. Conf. on Soil Mechanics and Foundation Engineering, vol. III, pp. 223-226, Tokyo 1977.

[16] LADE P.V. (1978) - "Prediction of undrained behavior of sand". J. of the Geotechnical Division. Proc. of the American Society of Civil Engineers. $n^{8}$ GT 6, pp. 723-736, June 1978.

[17] LADE P.V. (1975) - "Elasto-plastic stress-strain theory for cohesionless soil with curved yield surfaces". Report to the National Science Foundation University of California. Berkeley.

[18] MAIER G., HUECKEL T. (1977) - "Non associated and coupled flow rules of elasto-plasticity for geotechnical Media". Session Spéciale n 9, IX Int. Conf. on Soil Mechanics and Founfation Engineering. Tokyo (1977).

[19] MATSUOKA, H. (1974) - "Dilatancy characteristics of soils". Soils and foundations, Vol. 14, n³. pp. 13-24, Sept. 1974.
[20] SHOJI M., MATSUOTO T. (1976) - "Consolidation of embrankment foundation". Soils and foundations, vol. 16, n० 1, pp. 59-74, March 1976.

[21] MIR EMERATI (1969) - "Pression interstitielle dans les milieux poreux". Thèse Docteur-Ingénieur. Paris.

[22] MOH Z.C., BRAND E.W., NELSON J.D. (1972) "Pore pressure under a bund on soft fissured clay". Proceedings of the Purdue Conference on performance of Earth and Earth supported structures. American Society of Civil Engineers, Vol. I, pp. 243-272.

[23] MROZZ. (1969) - "An attempt to describe the behaviour of metals under cyclic loads using a more general work hardening model". Acta Mechanica, vol. 7, pp. 169212, 1969.

[24] NAYAK G.C., ZIENKIEWICZ O.C. (1972) - "Elastoplastic stress analysis. A generalization for various constitutive relations including strain soltening" Int. Journal of Num. Methods in Engineering, n 5, pp. 113-135, 1972.

[25] PILOT G. (1976) - "La stabilité des remblais sur sols mous" - Bulletin de liaison des Laboratoires des Ponts et Chaussées, numéro spécial "Stabilité des talus" II. pp. 83106, Déc. 1976.

[26] PREVOST J.H. (1977) - "Mathematical modelling of monotonic and cyclic undrained clay behaviour" Int. Journal Numer. Anal. Methods Geomech., vol. 1, pp. 195-216. 1977.

[27] SANGREY D.A. (1977) - "Response of Offshores piles to cyclic loading": Offshore Technology Conf. n²944, Houston, 1977.

[28] SKEMPTON A.W. (1954) - "The pore pressure coefficients $A$ and $B "$ ". Géotechnique, vol. 4, n० 4, December 1954.

[29] MATSUMOTO T. (1976) - "Finite element analysis of immediate and consolidation deformations based on effective stress principle". Soils and Foundations, vol. 16, n" 4. pp. - December 1976 .

[30] VUAILLAT P. (1979) - "Propriētés visqueuses d'une argile : expériences et formulation incrémentale". Thèse de docteur-ingénieur, Grenoble, à paraître en 1979. 